\title{
New Chitosan Polymer Scaffold Schiff Bases as Potential Cytotoxic Activity: Synthesis, Molecular Docking, and Physiochemical Characterization
}

\author{
Ponnusamy Packialakshmi ${ }^{1}$, Perumal Gobinath ${ }^{1}$, Daoud Ali ${ }^{2}$, Saud Alarifi ${ }^{2}$, \\ Raman Gurusamy ${ }^{3}$, Akbar ldhayadhulla ${ }^{1}$ and Radhakrishnan Surendrakumar ${ }^{1 *}$ \\ ${ }^{1}$ Research, Department of Chemistry, Nehru Memorial College, Affiliated Bharathidasan University, Puthanampatti, India, \\ ${ }^{2}$ Department of Zoology, College of Science, King Saud University (KSU), Riyadh, Saudi Arabia, ${ }^{3}$ Department of Life Sciences, \\ Yeungnam University, Gyeongsan, South Korea
}

\section{OPEN ACCESS}

Edited by:

Adnan Ahmed Bekhit, Alexandria University, Egypt

Reviewed by:

Ehsan Nazarzadeh Zare,

Damghan University, Iran Huawei He,

Southwest University, China

*Correspondence:

Radhakrishnan Surendrakumar surendrakumar@nmc.ac.in organicsurendar@gmail.com

Specialty section:

This article was submitted to Polymer Chemistry,

a section of the journal

Frontiers in Chemistry

Received: 17 October 2021 Accepted: 06 December 2021 Published: 17 January 2022

Citation: Packialakshmi P, Gobinath P, Ali D, Alarifi S, Gurusamy R, Idhayadhulla $A$ and Surendrakumar $R$ (2022) New Chitosan Polymer Scaffold Schiff Bases as Potential Cytotoxic Activity: Synthesis, Molecular Docking, and Physiochemical Characterization.

Front. Chem. 9:796599. doi: 10.3389/fchem.2021.796599
In this work, we synthesize the sulfonated Schiff bases of the chitosan derivatives 2a-2j without the use of a catalyst in two moderately straightforward steps with good yield within a short reaction time. The morphology and chemical structure of chitosan derivatives were investigated using FT-IR, NMR $\left({ }^{1} \mathrm{H}-{ }^{13} \mathrm{C}\right), \mathrm{XRD}$, and SEM. Furthermore, our chitosan derivatives were tested for their anticancer activity against the MCF-7 cancer cell line, and doxorubicin was used as a standard. In addition, the normal cell lines of the breast cancer cell MCF-10A, and of the lung cell MRC- 5 were tested. Compound $2 \mathrm{~h}$, with a $\mathrm{Gl}_{50}$ value of $0.02 \mu \mathrm{M}$ for MCF-7, is highly active compared with the standard doxorubicin and other compounds. The synthesized compounds 2a-2j exhibit low cytotoxicity, with $\mathrm{IC}_{50}>$ $100 \mu \mathrm{g} / \mathrm{ml}$, against normal cell lines MCF-10A, MRC-5. We also provide the results of an in-silico study involving the Methoxsalen protein (1Z11). Compound $2 \mathrm{~h}$ exhibits a higher binding affinity for $1 Z 11$ protein $(-5.9 \mathrm{kcal} / \mathrm{mol})$ and a lower binding affinity for Doxorubicin $(-5.3 \mathrm{kcal} / \mathrm{mol})$ than certain other compounds. As a result of the aforementioned findings, the use of compound $2 \mathrm{~h}$ has an anticancer drug will be researched in the future.

Keywords: schiff base, SEM, anticancer activity, MCF-7, in-silico molecular docking, structure activity relationship

\section{INTRODUCTION}

Cell proliferation becomes abnormal when it expands outside its normal limits, invades adjacent tissues, and/or feeds on other parts of the body, and can cause cancer. Cancer can begin anywhere in the body. A period of growth is the primary trigger for cancer-related death. The World Health Organization (WHO) reports that cancer remains the leading cause of death in most countries. Cancer is the second leading cause of death worldwide, with new cancer diagnoses expected to reach 18.1 million in 2018 and with deaths due to cancer expected to exceed 9.6 million. Both men and women can be affected by cancer. According to a UN DESA assessment from 2014, the world's population, currently at 7.3 billion people, will grow to 8.5 billion by 2030 , to 9.7 billion by 2050 , and to 11.2 billion by 2,100 . The estimate is that 1.5 billion cancer cases will bediagnosed, and that 1.2 billion fatalities will occur (Bray and Møller, 2006). In addition, with an anticipated 8.5 billion people on the planet, global mortality is expected to hit 2.14 billion by 2030 (Ali et al., 2011; Pereira et al., 2012). Cancers of the breast and lung are major causes of death in both women and men (5,22,000 deaths in 2012). In over 140 nations, these two cancers are the most often diagnosed (Ali et al., 2015). 
Lung cancer and female breast cancer are the two most lifethreatening malignancies, according to research. Breast cancer is one of the most frequent cancers in women and one of the most deadly (Bray et al., 2018). The activities of chitosan in killing cancer cells are known to work via the induction of apoptosis due to the activation of caspases 3,8, and 9; the modification of the $\mathrm{BaX}$ - to Bcl-2 ratio; and the induction of DNA damage (Adhikari and Yadav, 2018), with the features of chitosan protonation present in acidic environments. Chitosan and its derivatives have been shown to selectively permeate cancer cell membranes and exhibit anticancer activity via cellular enzymatic, antiangiogenic, immune-enhancing, antioxidant defense mechanism, and apoptotic mechanisms. This activity can be reduced in oral administration due to protonation of the amine group (Mahmood et al., 2019). Despite this, the clinical options for chitosan in the treatment of a number of human cancers remain limited (Kuppusamy and Karuppaiah, 2013). The therapeutic use of chitosan-based molecules with minimal noncancer cell damage is vital. Chitosan-based molecules were discovered to have anticancer properties that cause minimal damage to non-cancer cells (Wimardhani et al., 2014), and its effect varies greatly depending on the molecular weight and DDA against different cancer cell lines. Many cancer cells are affected by chemotherapeutic medicines such as cisplatin, 5- fluorouracil (5-FU), docetaxel, procarbazine, methotrexate, etc. according to studies conducted in vitro and in vivo (Andreadis et al., 2003). The Schiff base approach was used to make the DCMC/SS film, which has a wide range of biological applications including wound treatment, artificial skin, and tissue engineering (Wang et al., 2019). In addition to its mucoadhesive properties, chitosan suppresses drug efflux processes, allowing for greater drug penetration into cells (Taher et al., 2019). Chitosan and its derivatives, notably chitosan-drug nanocomposites as the principal anticancer formulations, can be viable natural choices to overcome these problems due to their selective antitumor effects, nontoxicity, biocompatibility, and biodegradability. Chitosan is a nontoxic, non-allergenic, biocompatible, and biodegradable natural polyaminosaccharide deacetylated from chitin (Imran et al., 2020). Chitosan has three types of reactive groups at C-2, $\mathrm{C}-3$, and C-6: the primary amine group, and the primary and secondary hydroxyl groups. Among the three reactive groups for chitosan's biological activity, glucosamine's main amine at C-2 is the most important group (Aranaz et al., 2010). Chitosan and chitosan compounds with higher DDA and lower MW had better antibacterial, antioxidant, and anticancer properties (Kim, 2018). Chitosan and its derivatives have good biological activity, including anticancer properties, and makes it a good carrier for the preparation of prodrugs, as an accelerator of tissue engineering, and as an excipient for drug delivery and gene delivery (Muanprasat and Chatsudthipong, 2017; Cheung et al., 2015; Zhang et al., 2002; Ahmed and Ikram, 2016; Zhang et al., 2018; Bakar et al., 2017). The biological characteristics of chitosan are influenced by its solubility in water and in other commonly used solvents (Kumirska et al., 2011). Under mild conditions, chitosan includes two types of reactive functional groups (an amino group and a hydroxyl group) that can be used to modify its characteristics and to generate other derivatives ( $\mathrm{Li}$ et al., 2015). Chitosan has garnered a lot of interest recently because of its commercial potential in the medical, chemical, and food industries. Chitosan is frequently employed in biomedical applications due to its ability to obtain an extremely adaptable combination of chemical elements and properties as well as due to its ease of extraction, chemical modifications, and low cost (Chen and Gong, 2016). Chitosan and chitosan derivatives have good anticancer effects and decrease the unwanted effects of some medicine due to its controlled administration of doses for cancer treatment and its slow release of the free drug from conjugates (Perni and Prokopovich, 2017). Following that, efforts are required to identify newer, more effective, and less toxic chemotherapeutic drugs for the treatment of cancers (Srivastava et al., 2019). The anticancer active compounds of chitosan analogs are shown in Figure 1 (Jiang et al., 2011; Adhikari and Yadav, 2018; Li et al., 2020; Saeed et al., 2020). In recent years, $\mathrm{CH}$-based tissue regeneration and drug delivery to implants as well as chitosan's potential for use as a biomaterial in dental applications have been researched (Husain et al., 2017; Qasim et al., 2018). Chitosan is utilized in the pharmaceutical industry for making drug delivery matrixes such as blend films (CS/PAH) as well as antibacterial activities (Ali et al., 2020; Sarwar et al., 2020).

The contemporary work addresses the development of the cytotoxic activity of fresh sulfonated Schiff base of chitosan derivatives can be synthesized and characterized by FT-IR, NMR $\left({ }^{1} \mathrm{H}\right.$ NMR, ${ }^{13} \mathrm{C}$ NMR) spectroscopy, and its physical properties were observed. In-silico molecular docking analysis and also evaluated the cytotoxic activities against a cancer cell lines.

\section{EXPERIMENTAL}

\section{Materials and Methods (Chemistry)}

All the substance and reagents such as chitosan (degree of deacetylation - 75\%, Mw141 KDa), acetic acid, AEEA, chlorosulfonicacid, aromatic aldehydes, ethanol and DMF are purchased from Merck. The melting points of synthesized substances were evaluated in open capillary tubes, and were uncorrected. The FT-IR spectra were recorded in $\mathrm{KBr}$ on a Shimadzu 8201pc $\left(4,000-1,000 \mathrm{~cm}^{-1}\right)$. The ${ }^{1} \mathrm{H}-\mathrm{NMR}$ and ${ }^{13} \mathrm{CNMR}$ spectra were recorded on a Bruker DRX-300 MHz. To obtain NMR spectra, the product was dissolved in DMSO$\mathrm{d}_{6}$. TLC was used to assess the purity of the compounds, with silica-gel and 60F254 aluminium sheets as the adsorbent.

\section{Preparation of Chitosan Analogue (1, 2, 2a-2j)}

Chitosan ( $3 \mathrm{~g}$, deacetylated) was dissolved in $20 \mathrm{ml}$ of $1 \%$ $\mathrm{CH}_{3} \mathrm{COOH} \quad(\mathrm{pH}=3.4-3.6)$ and aminoethylethanolamine (AEEA) $(2.0 \mathrm{ml})$ was reacted at $100^{\circ} \mathrm{C}$ for $4 \mathrm{~h}$ to obtain compound 1 . To adjacent the $\mathrm{pH}$ of compound $1,1 \mathrm{M} \mathrm{NaOH}$ was added. An electrophilic substitution process in which the proton was replaced by the $\mathrm{SO}_{3} \mathrm{H}$ group was the primary mechanism of the reaction (Morrison and Boyd, 1997). Compound 1 was dissolved in dimethylformamide (DMF), chlorosulfonic acid (4.5 ml in $30 \mathrm{ml} \mathrm{DMF})$ was fractionally 


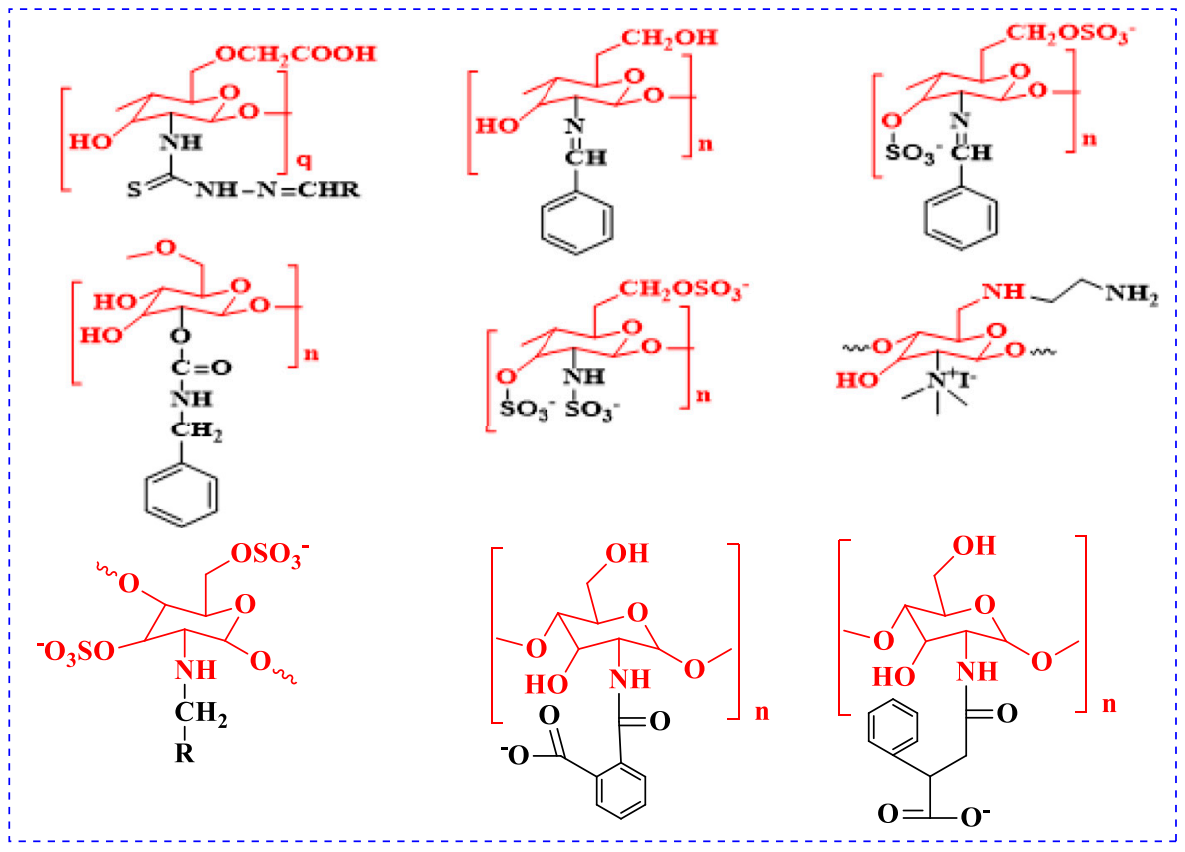

FIGURE 1 | Anticancer active compounds of chitosan derivatives.

added, and the mixture was stirred at room temperature for $5 \mathrm{~h}$. It was neutralized with a $20 \% \mathrm{NaOH}$ solution, and the precipitate of compound 2 was obtained, and reprecipitated with methanol twice (Martins et al., 2003). Compound $2(2.5 \mathrm{~g}$ dissolved in acetic acid with ethanol) and benzaldehyde $(1.0 \mathrm{ml})$ were mixed with ethanol and stirred for $8 \mathrm{~h}$ at $60^{\circ} \mathrm{C}$. The white gel obtained indicates the formation of Schiff base compound 2a. A solution of $5 \% \mathrm{NaOH}$ was used to precipitate the product, filtered, washed with ice-cold water, and add ethanol to remove any unreacted products. The final product was soluble in DMF. TLC was used to track the reaction's progress. In TLC, the eluting solvents were hexane and ethylacetate (4:6). Compounds $2 \mathrm{~b}-2 \mathrm{j}$ were synthesized using the technique outlined above.

\section{Synthesis of $(2,3,4,6)-3-($ benzylideneamino) -6-(((2-((2-hydroxyethyl)amino)ethyl)amino) methyl)-2,5-dimethoxytetrahydro- $2 \mathrm{H}$-pyran-4-yl sulfate (2a)}

Yield: 65\%;IR (KBr) $\left(\mathrm{cm}^{-1}\right)$; 3,452 (NH, str), 3,280 (OH-str), 2,951 (CH-str Ar ring), 1,645 ( $\mathrm{N}=\mathrm{CH}$, str), 1,383 (N-C, str), $1,326(\mathrm{~S}=\mathrm{O}), 972(\mathrm{C}-\mathrm{O}-\mathrm{S}, \mathrm{str}) .{ }^{1} \mathrm{H}$ NMR $\left(\mathrm{DMSO}_{-} \mathrm{d}_{6}\right), \delta(\mathrm{ppm})$ : $8.20(\mathrm{~s}, 1 \mathrm{H}, \mathrm{N}=\mathrm{CH}), 7.85-7.52(\mathrm{~m}, 5 \mathrm{H}, \mathrm{Ph}), 5.21-3.10(\mathrm{~m}, 5 \mathrm{H}$, $\mathrm{CS}-\mathrm{H}), 3.68(\mathrm{~s}, 1 \mathrm{H}, \mathrm{OH}), 3.52-3.33\left(\mathrm{~s}, 6 \mathrm{H}, \mathrm{OCH}_{3}-\mathrm{CS}\right)$, 3.45-2.70 (m, 4H, OH- $\left.\left(\mathrm{CH}_{2}\right)_{2}\right), 2.80-2.54\left(\mathrm{~m}, 2 \mathrm{H}, \mathrm{CH}_{2^{-}}\right.$ $\mathrm{NH}), 2.68-2.51\left(\mathrm{~m}, 4 \mathrm{H}, \mathrm{N}\left(\mathrm{CH}_{2}\right)_{2}\right), 2.02(\mathrm{~s}, 2 \mathrm{H}, \mathrm{NH}) ;{ }^{13} \mathrm{C}$ NMR (DMSO- $\left.\mathrm{d}_{6}\right) \delta(\mathrm{ppm}): 163.8(1 \mathrm{C}, \mathrm{N}=\mathrm{CH}), 136.5,131.2$, $129.3,128.9$ (6C, Ph), 112.3, 87.4, 71.5, 65.2, 60.7 (5C, CS), 61.7, 52.0 (2C, C-OH), 57.6, 55.7 (2C, $\left.\mathrm{OCH}_{3}-\mathrm{CS}\right), 49.6,49.0$ (2C, N-C in amine), 48.4 (1C, N-CH).

\section{Synthesis of $(2,3,4,6)-3-((4-c h l o r o b e n z y$ lidene)amino)-6-(((2-((2-hydroxyethyl) amino)ethyl) amino)methyl)- 2,5-dimethoxytetrahydro- 2H-pyran-4-ylsulfate (2b)}

Yield: 68\%; IR (KBr) (cm $\left.{ }^{-1}\right)$; 3,454 (NH, str), 3,286 (OH-str), 2,954 (CH-str Ar ring), 1,641 ( $\mathrm{N}=\mathrm{CH}$, str), 1,378 (N-C, str), 1,320 ( $\mathrm{S}=$ O), 972 (C-O-S, str), 544 (C-Cl). ${ }^{1} \mathrm{H}$ NMR (DMSO-d $), \delta$ (ppm): $8.18(\mathrm{~s}, 1 \mathrm{H}, \mathrm{N}=\mathrm{CH}), 7.80-7.50(\mathrm{~m}, 4 \mathrm{H}, \mathrm{Cl}-\mathrm{Ph}), 5.23-3.12(\mathrm{~m}, 5 \mathrm{H}$, $\mathrm{CS}-\mathrm{H}), 3.66$ (s, 1H, OH), 3.54-3.35 (s, 6H, $\left.\mathrm{OCH}_{3}-\mathrm{CS}\right), 3.47-2.72$ $\left(\mathrm{m}, 4 \mathrm{H}, \mathrm{OH}-\left(\mathrm{CH}_{2}\right)_{2}\right), 2.82-2.56\left(\mathrm{~m}, 2 \mathrm{H}, \mathrm{CH}_{2}-\mathrm{NH}\right), 2.65-2.50(\mathrm{~m}$, $\left.4 \mathrm{H}, \mathrm{N}\left(\mathrm{CH}_{2}\right)_{2}\right), 2.04$ (s, $\left.2 \mathrm{H}, \mathrm{NH}\right) ;{ }^{13} \mathrm{C}$ NMR $\left(\mathrm{DMSO}_{-} \mathrm{d}_{6}\right) \delta$ (ppm): $163.4(1 \mathrm{C}, \mathrm{N}=\mathrm{CH}), 136.8,134.2,130.3,129.0$ (6C,Cl- $\mathrm{Ph}), 112.5$, 87.6, 71.7, 65.4, 60.9 (5C, CS), 61.9, 52.2 (2C, C-OH), 57.8, 55.9 (2C, $\left.\mathrm{OCH}_{3}-\mathrm{CS}\right), 49.8,49.2$ (2C, N-C in amine), 48.6 (1C, N-CH).

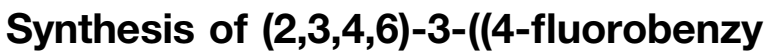 lidene)amino)-6-(((2-)((2-hydroxyethyl) amino)ethyl)amino)methyl)- 2,5-dimethoxytetrahydro- 2H-pyran-4-ylsulfate (2c)}

Yield: 70\%; IR (KBr) $\left(\mathrm{cm}^{-1}\right)$; 3,450 (NH, str), 3,282 (OH-str), 2,945 (CH-str Ar ring), 1,643 ( $\mathrm{N}=\mathrm{CH}$, str), 1,384 (N-C, str),

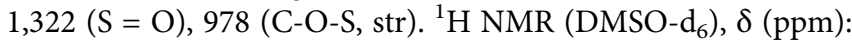
8.41 (s, $1 \mathrm{H}, \mathrm{N}=\mathrm{CH}), 7.85-7.38(\mathrm{~m}, 4 \mathrm{H}, \mathrm{F}-\mathrm{Ph}), 5.20-3.08(\mathrm{~m}, 5 \mathrm{H}$, $\mathrm{CS}-\mathrm{H}), 3.65$ (s, $1 \mathrm{H}, \mathrm{OH}), 3.50-3.31$ (s, $\left.6 \mathrm{H}, \mathrm{OCH}_{3}-\mathrm{CS}\right), 3.43-2.68$ (m, $\left.4 \mathrm{H}, \mathrm{OH}-\left(\mathrm{CH}_{2}\right)_{2}\right), 2.81-2.53\left(\mathrm{~m}, 2 \mathrm{H}, \mathrm{CH}_{2}-\mathrm{NH}\right), 2.66-2.52(\mathrm{~m}$, $\left.4 \mathrm{H}, \mathrm{N}\left(\mathrm{CH}_{2}\right)_{2}\right), 2.03$ (s, $\left.2 \mathrm{H}, \mathrm{NH}\right) ;{ }^{13} \mathrm{C}$ NMR $\left(\mathrm{DMSO}_{-} \mathrm{d}_{6}\right) \delta(\mathrm{ppm})$ : 165.5, 132.2, 130.3, 115.9 (6C, F-Ph), $163.6(1 \mathrm{C}, \mathrm{N}=\mathrm{CH}), 112.1$, 
<smiles>COC1C(N)C(O)C(OC)C1O</smiles>

Step-1

Aceticacid AEEA, $4 \mathrm{hr}$ $100^{\circ} \mathrm{C}$
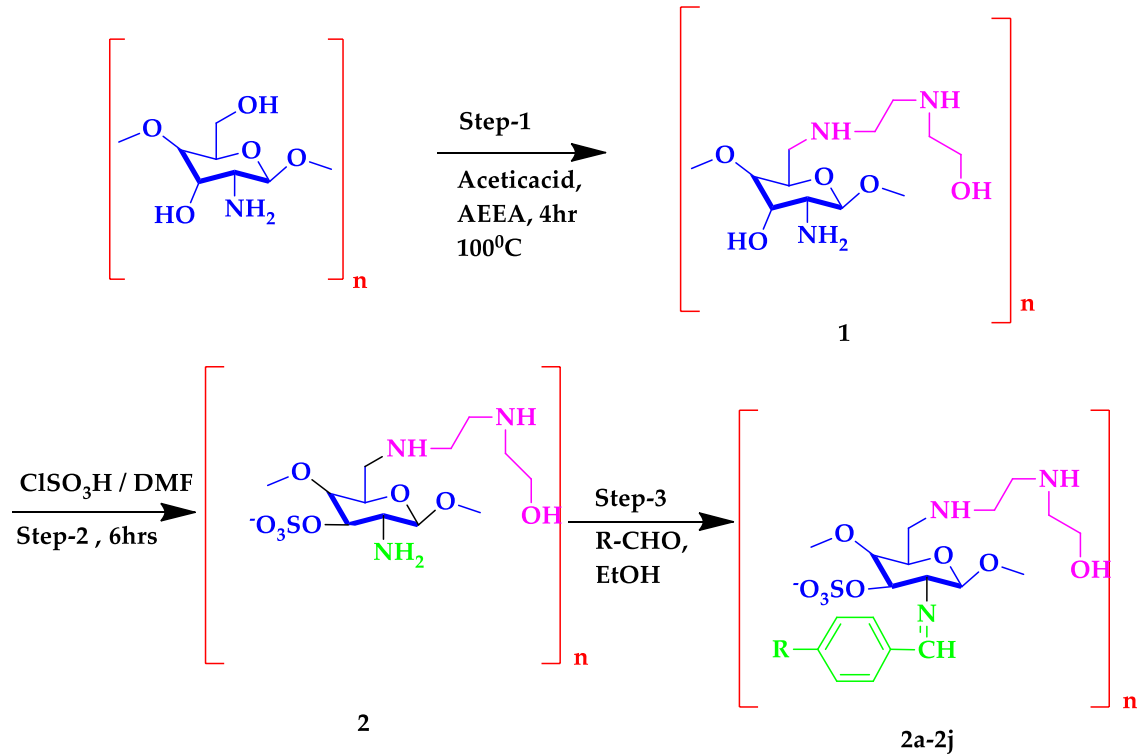

$\mathrm{R}=\mathrm{C}_{6} \mathrm{H}_{5}-\mathrm{CHO}, 4-\mathrm{Cl}-\mathrm{C}_{6} \mathrm{H}_{4}, 4-\mathrm{F}-\mathrm{C}_{6} \mathrm{H}_{4}, 4-\mathrm{OH}-\mathrm{C}_{6} \mathrm{H}_{4}, 4-\mathrm{OCH}_{3}-\mathrm{C}_{6} \mathrm{H}_{4}, 4-\mathrm{N}\left(\mathrm{CH}_{3}\right) 2-\mathrm{C}_{6} \mathrm{H}_{4}$, $4-\mathrm{NO}_{2}-\mathrm{C}_{6} \mathrm{H}_{4}, 4-\mathrm{OH}-3-\mathrm{OCH}_{3}-\mathrm{C}_{6} \mathrm{H}_{3}, 2-\mathrm{OH}-\mathrm{C}_{6} \mathrm{H}_{4}$, Furfural.

SCHEME 1 | The synthesized Schiff base of sulfonated chitosan derivatives 2a-2j.

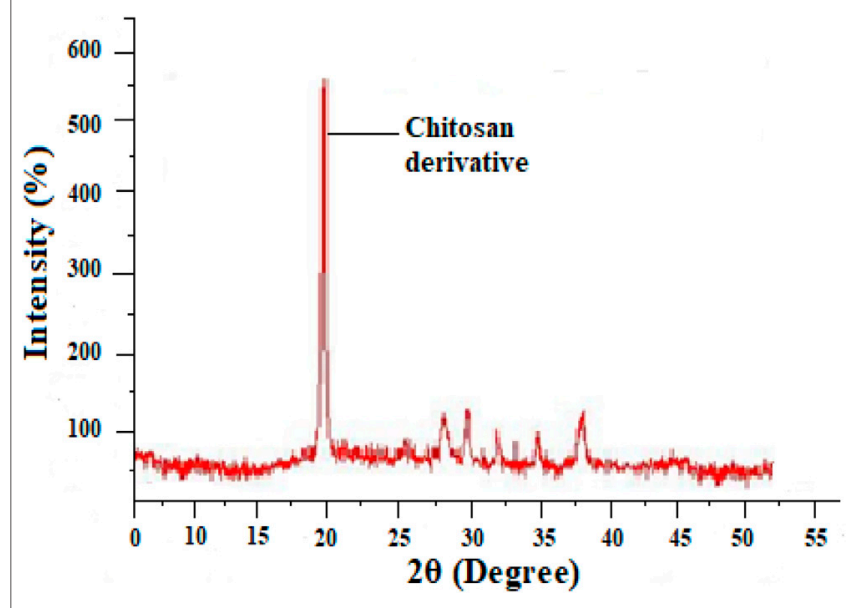

FIGURE 2 | X-ray diffraction study of chitosan derivative.

87.3, 71.2, 65.0, 60.5 (5C, CS), 61.4, 52.1 (2C, C-OH), 57.4, 55.3 (2C, $\left.\mathrm{OCH}_{3}-\mathrm{CS}\right), 49.7,49.1$ (2C, N-C in amine), 48.2 (1C, N-CH).

\section{Synthesis of $(2,3,4,6)-3-((4-h y d r o x y b e n z y l$} idene)amino)-6-(((2-((2-hydroxyethyl)amino) ethyl)amino)methyl)-2,5-dimethoxytetra hydro-2H-pyran-4-ylsulfate (2d)

Yield: 62\%; IR (KBr) $\left(\mathrm{cm}^{-1}\right)$; 3,458 (NH, str), 3,285 (OH-str), 2,956 (CH-str Ar ring), 1,643 ( $\mathrm{N}=\mathrm{CH}$, str), 1,385 (N-C, str), 1,327 ( $\mathrm{S}=\mathrm{O})$, 980 (C-O-S, str). ${ }^{1} \mathrm{H}$ NMR (DMSO-d 6 ), $\delta$ (ppm): 10.54 (s, 1H, OH$\mathrm{Ph}), 8.61(\mathrm{~s}, 1 \mathrm{H}, \mathrm{N}=\mathrm{CH}), 7.80-6.82(\mathrm{~m}, 4 \mathrm{H}, \mathrm{OH}-\mathrm{Ph}), 5.22-3.11(\mathrm{~m}$, $5 \mathrm{H}, \mathrm{CS}-\mathrm{H}), 3.69$ (s, $1 \mathrm{H}, \mathrm{OH}), 3.53-3.34$ (s, $\left.6 \mathrm{H}, \mathrm{OCH}_{3}-\mathrm{CS}\right), 3.46-2.71$ (m, 4H, OH- $\left.\left(\mathrm{CH}_{2}\right)_{2}\right), 2.81-2.53\left(\mathrm{~m}, 2 \mathrm{H}, \mathrm{CH}_{2}-\mathrm{NH}\right), 2.69-2.52(\mathrm{~m}, 4 \mathrm{H}$, $\left.\mathrm{N}\left(\mathrm{CH}_{2}\right)_{2}\right), 2.03(\mathrm{~s}, 2 \mathrm{H}, \mathrm{NH}) ;{ }^{13} \mathrm{C}$ NMR $\left(\mathrm{DMSO}_{-} \mathrm{d}_{6}\right) \delta$ (ppm): 163.9 $(1 \mathrm{C}, \mathrm{N}=\mathrm{CH}), 160.5,130.2,129.3,116.9(6 \mathrm{C}, \mathrm{OH}-\mathrm{Ph}), 112.2,87.3,71.4$, 65.3, 60.6 (5C, CS), 61.6, 52.1 (2C, C-OH), 57.5, 55.6 (2C, $\left.\mathrm{OCH}_{3}-\mathrm{CS}\right)$, 49.7, 49.1 (2C, N-C in amine), $48.3(1 \mathrm{C}, \mathrm{N}-\mathrm{CH})$.

\section{Synthesis of $(2,4,5,6)-2-(((2-)((2-$} hydroxyethyl)amino)ethyl)amino)

\section{methyl)-3,6-dimethoxy-} 5-((4-methoxybenzylidene)amino) tetrahydro-2H-pyran-4-yl sulfate (2e)

Yield: 67\%; IR (KBr) $\left(\mathrm{cm}^{-1}\right)$; 3,455 (NH, str), 3,287 (OH-str), 2,952 (CH-str Ar ring), 1,646 ( $\mathrm{N}=\mathrm{CH}, \mathrm{str}), 1,386(\mathrm{~N}-\mathrm{C}$, str), 1,328 ( $=\mathrm{O}), 982(\mathrm{C}-\mathrm{O}-\mathrm{S}, \mathrm{str}) .{ }^{1} \mathrm{H}$ NMR $\left(\mathrm{DMSO}_{6} \mathrm{~d}_{6}\right), \delta(\mathrm{ppm})$ : 8.45 (s, $1 \mathrm{H}, \mathrm{N}=\mathrm{CH}), 7.89-7.10\left(\mathrm{~m}, 4 \mathrm{H}, \mathrm{OCH}_{3}-\mathrm{Ph}\right), 5.25-3.14(\mathrm{~m}$, $5 \mathrm{H}, \mathrm{CS}-\mathrm{H}), 3.86\left(\mathrm{~s}, 3 \mathrm{H}, \mathrm{OCH}_{3}\right), 3.70(\mathrm{~s}, 1 \mathrm{H}, \mathrm{OH}), 3.55-3.36(\mathrm{~s}$, $\left.6 \mathrm{H}, \mathrm{OCH}_{3}-\mathrm{CS}\right), 3.48-2.74\left(\mathrm{~m}, 4 \mathrm{H}, \mathrm{OH}-\left(\mathrm{CH}_{2}\right)_{2}\right), 2.84-2.57(\mathrm{~m}$, 2H, $\left.\mathrm{CH}_{2}-\mathrm{NH}\right), 2.69-2.55\left(\mathrm{~m}, 4 \mathrm{H}, \mathrm{N}\left(\mathrm{CH}_{2}\right)_{2}\right), 2.06$ (s, 2H, NH); ${ }^{13} \mathrm{C}$ NMR (DMSO-d 6 ) $\delta(\mathrm{ppm}): 163.4(1 \mathrm{C}, \mathrm{N}=\mathrm{CH}), 162.5,130.4$, $128.3,114.6\left(6 \mathrm{C}, \mathrm{OCH}_{3}-\mathrm{Ph}\right), 112.6,87.7,71.8,65.5,60.9$ (5C, CS), 61.4, 52.3 (2C, C-OH), 57.9, 55.8 (2C, $\left.\mathrm{OCH}_{3}-\mathrm{CS}\right), 55.4$ (1C, $\left.\mathrm{OCH}_{3}-\mathrm{Ph}\right), 49.9,49.1$ (2C, N-C in amine), 48.5 (1C, N-CH).

\section{Synthesis of $(2,3,4,6)-3-((4-($ dimethylamino)} benzylidene)amino)-6-(( 2 ((2- hydroxyethyl) amino)ethyl)amino) methyl)-2,5-dimethoxytetrahydro-2Hpyran-4-ylsulfate (2f)

Yield: 72\%; IR (KBr) $\left(\mathrm{cm}^{-1}\right)$; 3,457 (NH, str), 3,286 (OH-str), 1944 (CH-str Ar ring), 1,645 ( $\mathrm{N}=\mathrm{CH}$, str), 1,386 (N-C, str), 1,329 


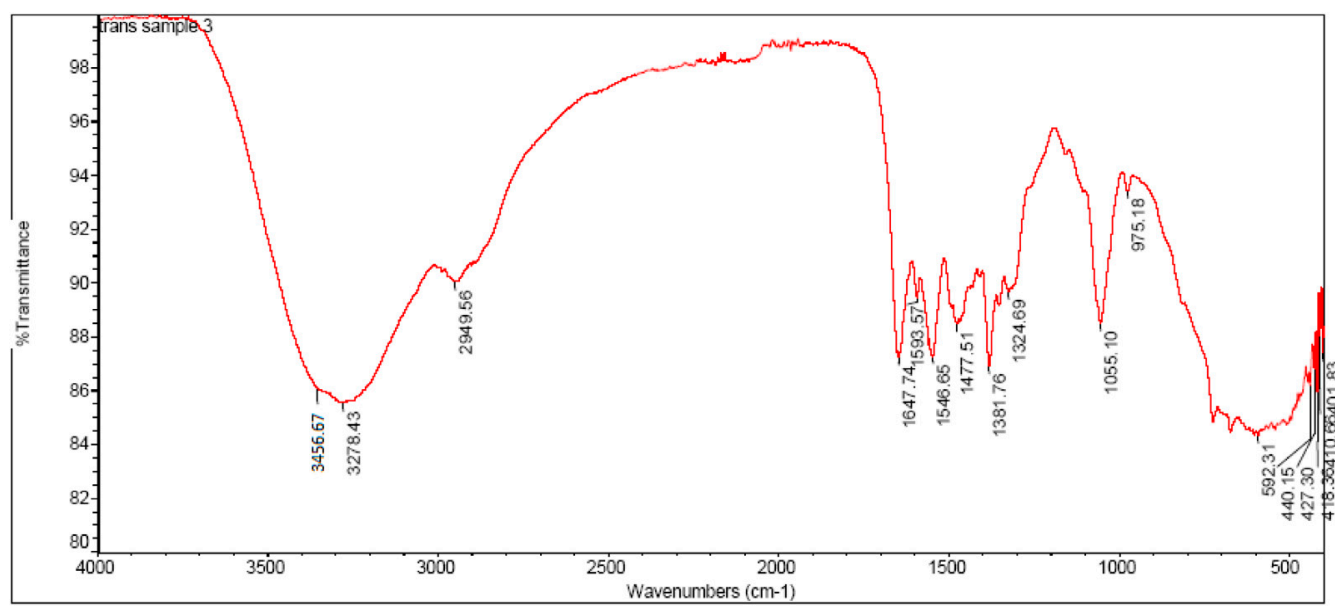

FIGURE 3 | FTIR Spectrum of compound-2h.

$(\mathrm{S}=\mathrm{O}), 980\left(\mathrm{C}-\mathrm{O}-\mathrm{S}\right.$, str). ${ }^{1} \mathrm{H}$ NMR (DMSO-d $\left.{ }_{6}\right), \delta(\mathrm{ppm}): 8.27(\mathrm{~s}$, $1 \mathrm{H}, \mathrm{N}=\mathrm{CH}), 7.55-6.86\left(\mathrm{~m}, 4 \mathrm{H}, \mathrm{N}\left(\mathrm{CH}_{3}\right)_{2}-\mathrm{Ph}\right), 5.25-3.14(\mathrm{~m}, 5 \mathrm{H}$, CS-H), 3.70 (s, 1H, OH), 3.56-3.37 (s, 6H, $\left.\mathrm{OCH}_{3}-\mathrm{CS}\right), 3.47-2.73$ $\left(\mathrm{m}, 4 \mathrm{H}, \mathrm{OH}-\left(\mathrm{CH}_{2}\right)_{2}\right), 3.05\left(\mathrm{~s}, 6 \mathrm{H}, \mathrm{N}\left(\mathrm{CH}_{3}\right)_{2}\right), 2.82-2.56(\mathrm{~m}, 2 \mathrm{H}$, $\left.\mathrm{CH}_{2}-\mathrm{NH}\right), 2.64-2.50\left(\mathrm{~m}, 4 \mathrm{H}, \mathrm{N}\left(\mathrm{CH}_{2}\right)_{2}\right), 2.06(\mathrm{~s}, 2 \mathrm{H}, \mathrm{NH}) ;{ }^{13} \mathrm{C}$ NMR (DMSO- $\left.\mathrm{d}_{6}\right) \delta(\mathrm{ppm}): 163.5(1 \mathrm{C}, \mathrm{N}=\mathrm{CH}), 153.4,126.0$, 124.3, 112.0, 41.5 (8C, $\left.\mathrm{N}\left(\mathrm{CH}_{3}\right)_{2}-\mathrm{Ph}\right), 112.5,87.6,71.7,65.4,60.3$ (5C, CS), 61.8, 52.4 (2C, C-OH), 57.8, 55.4 (2C, $\left.\mathrm{OCH}_{3}-\mathrm{CS}\right), 49.8$, 49.1 (2C, N-C in amine), $48.6(1 \mathrm{C}, \mathrm{N}-\mathrm{CH})$.

\section{Synthesis of $(2,4,5,6)-2-(((2-)((2-h y d r o x y$ ethyl)amino)ethyl)amino)methyl)- 3,6-dimethoxy-5-((4-nitrobenzylidene)} amino)tetrahydro-2H-pyran-4-yl sulfate (2g) Yield: 76\%;IR (KBr) $\left(\mathrm{cm}^{-1}\right)$; 3,459 (NH, str), 3,285 (OH-str), 2,951 (CH-str Ar ring), 1,641 ( $\mathrm{N}=\mathrm{CH}$, str), 1,474 (N-O, str),

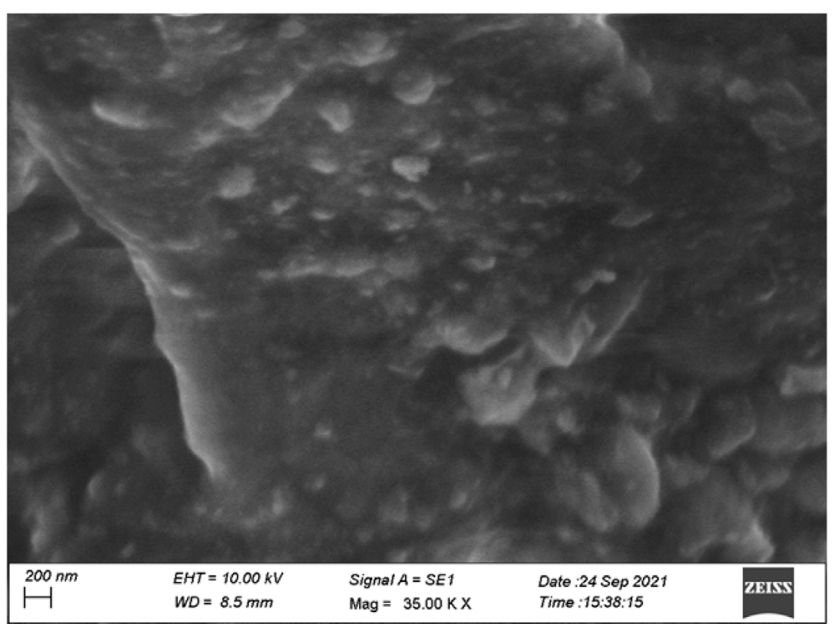

FIGURE 4 | SEM image of chitosan derivative.
1,385 (N-C, str), 1,321 ( $\mathrm{S}=\mathrm{O}), 973$ (C-O-S, str). ${ }^{1} \mathrm{H}$ NMR (DMSO-d $\left.\mathrm{d}_{6}\right), \delta(\mathrm{ppm}): 8.26(\mathrm{~s}, 1 \mathrm{H}, \mathrm{N}=\mathrm{CH}), 8.43-8.12(\mathrm{~m}, 4 \mathrm{H}$, $\left.\mathrm{NO}_{2}-\mathrm{Ph}\right), 5.23-3.12(\mathrm{~m}, 5 \mathrm{H}, \mathrm{CS}-\mathrm{H}), 3.64(\mathrm{~s}, 1 \mathrm{H}, \mathrm{OH}), 3.55-3.35$ (s, 6H, $\left.\mathrm{OCH}_{3}-\mathrm{CS}\right), 3.47-2.73\left(\mathrm{~m}, 4 \mathrm{H}, \mathrm{OH}-\left(\mathrm{CH}_{2}\right)_{2}\right), 2.82-2.53$ (m, $\left.2 \mathrm{H}, \mathrm{CH}_{2}-\mathrm{NH}\right), 2.65-2.52\left(\mathrm{~m}, 4 \mathrm{H}, \mathrm{N}\left(\mathrm{CH}_{2}\right)_{2}\right), 2.06(\mathrm{~s}, 2 \mathrm{H}$, $\mathrm{NH}) ;{ }^{13} \mathrm{C} \mathrm{NMR}\left(\mathrm{DMSO}-\mathrm{d}_{6}\right) \delta(\mathrm{ppm}): 163.3(1 \mathrm{C}, \mathrm{N}=\mathrm{CH}), 150.5$, $142.2,130.3,124.9\left(6 \mathrm{C}, \mathrm{NO}_{2} \mathrm{Ph}\right), 112.4,87.6,71.3,65.5,60.4$ (5C, CS), 61.3, 52.4 (2C, C-OH), 57.4, 55.3 (2C, $\left.\mathrm{OCH}_{3}-\mathrm{CS}\right), 49.7$, 49.2 (2C, N-C in amine), 48.5 (1C, N-CH).

\section{Synthesis of $(2,3,4,6)-3-((4-h y d r o x y-$ 3-methoxybenzylidene)amino)-6-((2- ((2-hydroxyethyl) amino)ethyl)amino) methyl)-2,5-dimethoxytetrahydro- 2H-pyran-4-ylsulfate (2h)}

Yield: 84\%; IR (KBr) $\left(\mathrm{cm}^{-1}\right)$; 3,456 (NH, str), 3,278 (OH-str), 2,949 (CH-str Ar ring), 1,647 ( $\mathrm{N}=\mathrm{CH}, \mathrm{str}), 1,381$ (N-C, str), 1,324 ( $\mathrm{S}=\mathrm{O}, \mathrm{str}), 975$ (C-O-S, str). ${ }^{1} \mathrm{H}$ NMR (DMSO-d $\left.\mathrm{d}_{6}\right), \delta$

\begin{tabular}{lccc}
\hline \multicolumn{3}{l}{ TABLE 1 | Anticancer activity of compounds $(\mu \mathrm{M})(2 \mathrm{a}-2 \mathrm{j})}$. \\
\hline Compounds & \multicolumn{3}{c}{ MCF-7 cell line } \\
\cline { 2 - 4 } & $\mathbf{G I}_{\mathbf{5 0}}(\boldsymbol{\mu M})$ & TGI $(\boldsymbol{\mu M})$ & $\mathbf{L C}_{\mathbf{5 0}}(\boldsymbol{\mu M})$ \\
\hline $2 \mathrm{a}$ & $03.3 \pm 0.23$ & $07.3 \pm 1.15$ & $07.5 \pm 0.03$ \\
$2 \mathrm{~b}$ & $0.05 \pm 0.15$ & $0.65 \pm 2.45$ & $0.89 \pm 0.28$ \\
$2 \mathrm{c}$ & $0.20 \pm 0.82$ & $0.50 \pm 1.50$ & $0.75 \pm 0.35$ \\
$2 \mathrm{~d}$ & $0.10 \pm 0.74$ & $0.70 \pm 0.68$ & $1.50 \pm 1.40$ \\
$2 \mathrm{e}$ & $0.05 \pm 0.33$ & $0.06 \pm 1.32$ & $0.79 \pm 1.25$ \\
$2 \mathrm{f}$ & $0.65 \pm 0.10$ & $11.0 \pm 1.62$ & $20.3 \pm 0.24$ \\
$2 \mathrm{~g}$ & $0.43 \pm 0.90$ & $0.75 \pm 1.53$ & $15.6 \pm 0.72$ \\
$2 \mathrm{~h}$ & $0.02 \pm 0.24$ & $0.15 \pm 0.19$ & $0.72 \pm 0.31$ \\
$2 \mathrm{i}$ & $0.07 \pm 0.56$ & $0.60 \pm 0.41$ & $2.25 \pm 0.31$ \\
$2 \mathrm{j}$ & $0.04 \pm 0.50$ & $0.29 \pm 0.72$ & $9.12 \pm 1.21$ \\
Doxorubicin & $0.04 \pm 0.75$ & $0.25 \pm 0.23$ & $0.80 \pm 0.46$
\end{tabular}
experiments. 
TABLE 2 | In vitro cytotoxicity of chitosan derivatives (2a-2j) on normal cells.

\begin{tabular}{lcc}
\hline Compd. No & MCF-10A & MRC5 \\
\cline { 1 - 1 }- & IC50 $(\boldsymbol{\mu M})$ & IC50 $(\boldsymbol{\mu M})$ \\
\hline $2 \mathrm{a}$ & 65.62 & 60.53 \\
$2 \mathrm{~b}$ & 72.04 & 75.35 \\
$2 \mathrm{c}$ & 71.51 & 73.03 \\
$2 \mathrm{~d}$ & 54.16 & 50.37 \\
$2 \mathrm{e}$ & 62.08 & 67.59 \\
$2 \mathrm{f}$ & 56.80 & 51.11 \\
$2 \mathrm{~g}$ & 74.62 & 70.23 \\
$2 \mathrm{~h}$ & 86.5 & 80.84 \\
$2 \mathrm{i}$ & 69.04 & 58.95 \\
$2 \mathrm{j}$ & 52.46 & 61.27
\end{tabular}

Each compound was tested in triplicate. All error bars represent mean $\pm S D$ from three independent experiments.

(ppm): 8.20 (s, 1H, N = CH), 7.55-6.93 (m, 3H, Vanillin), 5.37 (s, $1 \mathrm{H}, \mathrm{OH}-\mathrm{Ph}), 5.20-3.13$ (m, 5H, CS-H), 3.63 (s, 1H, OH), 3.73 (s, $\left.3 \mathrm{H}, \mathrm{OCH}_{3}\right), 3.50-3.31$ (s, $\left.6 \mathrm{H}, \mathrm{OCH}_{3}-\mathrm{CS}\right), 3.42-2.68(\mathrm{~m}, 4 \mathrm{H}, \mathrm{OH}-$ $\left.\left(\mathrm{CH}_{2}\right)_{2}\right), 2.83-2.53\left(\mathrm{~m}, 2 \mathrm{H}, \mathrm{CH}_{2}-\mathrm{NH}\right), 2.65-2.52(\mathrm{~m}, 4 \mathrm{H}, \mathrm{N}$ $\left.\left(\mathrm{CH}_{2}\right)_{2}\right), 2.07(\mathrm{~s}, 2 \mathrm{H}, \mathrm{NH}) ;{ }^{13} \mathrm{C} \mathrm{NMR}\left(\mathrm{DMSO}-\mathrm{d}_{6}\right) \delta(\mathrm{ppm})$ : $163.4(1 \mathrm{C}, \mathrm{N}=\mathrm{CH}), 151.5,149.2,133.3,123.0,117.2,112.5$ (6C, Vanillin), 112.4, 87.1, 71.3, 65.0, 60.4 (5C, CS), 61.6, 52.2 (2C, $\mathrm{C}-\mathrm{OH}), 57.5,55.4\left(2 \mathrm{C}, \mathrm{OCH}_{3}-\mathrm{CS}\right), 56.5\left(1 \mathrm{C}, \mathrm{OCH}_{3}\right), 49.7,49.1$ (2C, N-C in amine), $48.2(1 \mathrm{C}, \mathrm{N}-\mathrm{CH})$.

\section{Synthesis of $(2,3,4,6)-3-((2-h y d r o x y$ benzylidene)amino)-6-(((2-((2- hydroxyethyl) amino)ethyl) amino)methyl)-2,5-dimethoxy tetrahydro-2H-pyran-4-yl sulfate (2i)}

Yield: 73\%; IR (KBr) $\left(\mathrm{cm}^{-1}\right)$; 3,458 (NH, str), 3,284 (OH-str), 2,950 (CH-str Ar ring), 1,642 ( $\mathrm{N}=\mathrm{CH}$, str), 1,386 (N-C, str), $1,326(\mathrm{~S}=\mathrm{O}), 989(\mathrm{C}-\mathrm{O}-\mathrm{S}, \mathrm{str}) .{ }^{1} \mathrm{H}$ NMR $\left(\mathrm{DMSO}_{-} \mathrm{d}_{6}\right), \delta(\mathrm{ppm})$ : 8.27 (s, $1 \mathrm{H}, \mathrm{N}=\mathrm{CH}), 7.68-7.03(\mathrm{~m}, 4 \mathrm{H}, \mathrm{Ph}), 5.45$ (s, 1H, OH-Ph), 5.24-3.13 (m, 5H, CS-H), $3.65(\mathrm{~s}, 1 \mathrm{H}, \mathrm{OH}), 3.54-3.35(\mathrm{~s}, 6 \mathrm{H}$,

TABLE 3 | Molecular docking interaction of compounds 2a-2j and Doxorubicin against protein $1 Z 11$.

Compound Human microsomal cytochrome P450 2A6 complexed with methoxsalen (PDB ID: 1Z11)

\begin{tabular}{lccl}
\cline { 2 - 3 } & $\begin{array}{c}\text { Binding affinity } \\
\text { (kcal/mol) }\end{array}$ & $\begin{array}{c}\text { No. of } \\
\text { H-bonds }\end{array}$ & H-bonding residues \\
\hline $2 \mathrm{a}$ & -5.6 & 1 & Arg373 \\
$2 \mathrm{~b}$ & -5.0 & 2 & Asp108 \\
$2 \mathrm{c}$ & -5.2 & 2 & Gly113 and Arg373 \\
$2 \mathrm{~d}$ & -4.8 & 1 & Asn120 \\
$2 \mathrm{e}$ & -4.4 & 3 & Trp109, Asn120 and \\
& & & Lys228 \\
$2 \mathrm{f}$ & -5.7 & 2 & Phe118 and Asn120 \\
$2 \mathrm{~g}$ & -4.8 & 2 & Phe118 and Asn120 \\
$2 \mathrm{~h}$ & -5.9 & 2 & Phe118 and Asn120 \\
$2 \mathrm{i}$ & -4.8 & 2 & Phe118 and Asn120 \\
$2 \mathrm{j}$ & -5.2 & 3 & Asn120 and Arg373 \\
Doxorubicin & -5.3 & 1 & Arg373 \\
& & &
\end{tabular}

$\left.\mathrm{OCH}_{3}-\mathrm{CS}\right), 3.43-2.74\left(\mathrm{~m}, 4 \mathrm{H}, \mathrm{OH}-\left(\mathrm{CH}_{2}\right)_{2}\right), 2.83-2.55(\mathrm{~m}, 2 \mathrm{H}$, $\left.\mathrm{CH}_{2}-\mathrm{NH}\right), 2.63-2.52\left(\mathrm{~m}, 4 \mathrm{H}, \mathrm{N}\left(\mathrm{CH}_{2}\right)_{2}\right), 2.06(\mathrm{~s}, 2 \mathrm{H}, \mathrm{NH}) ;{ }^{13} \mathrm{C}$ NMR (DMSO-d $\left.\mathrm{d}_{6}\right) \delta(\mathrm{ppm}): 163.6(1 \mathrm{C}, \mathrm{N}=\mathrm{CH}), 161.5,132.6$, 132.0, 124.9, 124.0 (6C, Ph), 112.1, 87.2, 71.5, 65.3, 60.4 (5C, CS), 61.5, 52.6 (2C, C-OH), 57.7, 55.8 (2C, $\left.\mathrm{OCH}_{3}-\mathrm{CS}\right), 49.9,49.0$ (2C, $\mathrm{N}-\mathrm{C}$ in amine), 48.5 (1C, N-CH).

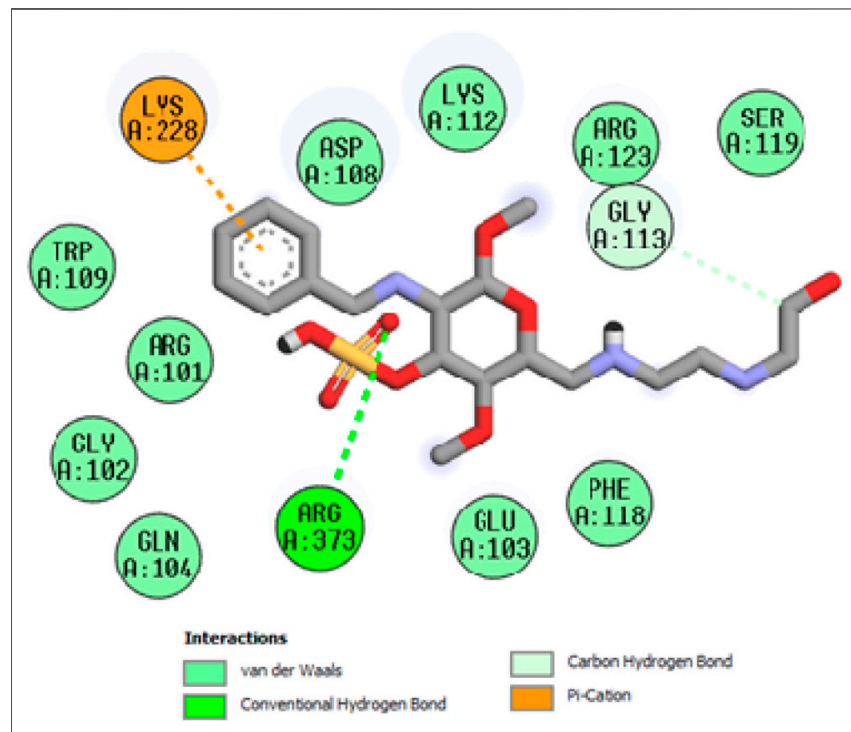

FIGURE 5 | Interaction of compound 2a in the binding pocket of 1211 receptor.

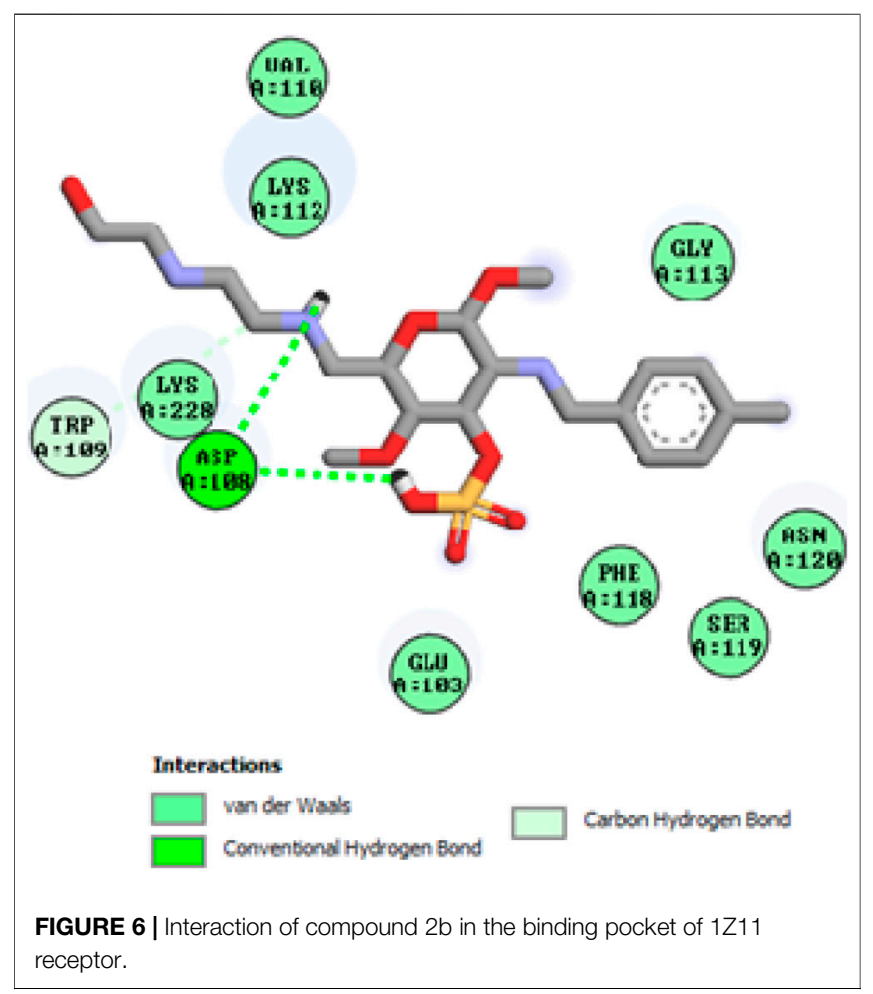




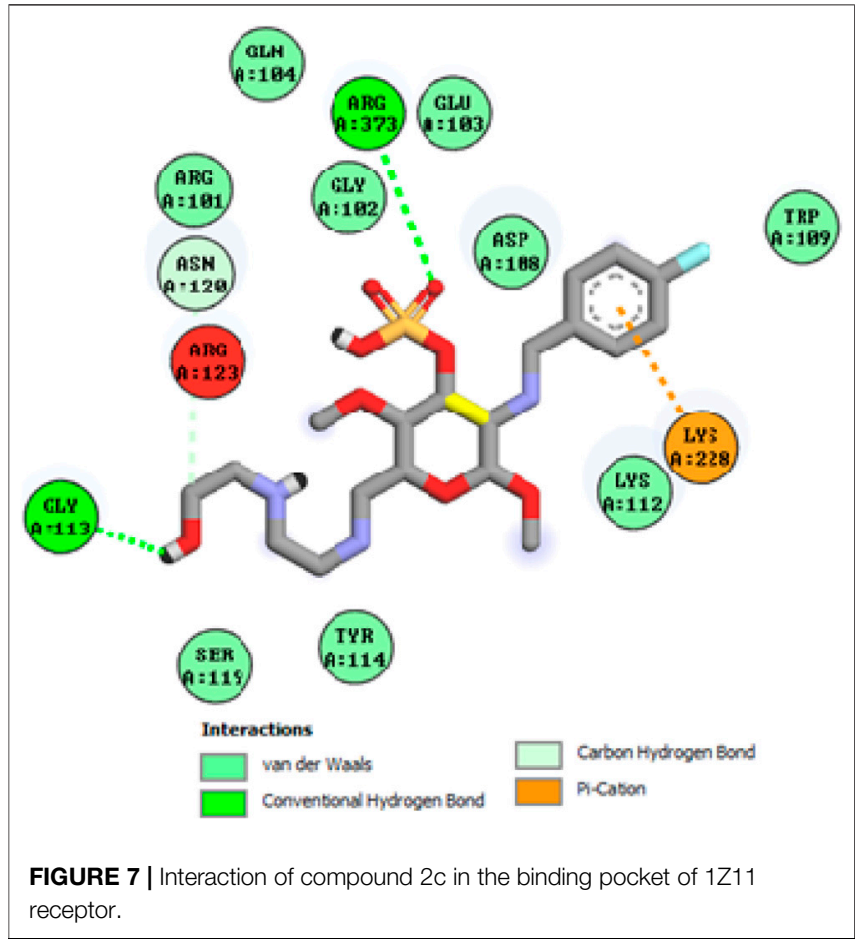

Synthesis of $(2,3,4,6)-3-(($ furan-2-ylmethy lene)amino)-6-(((2-((2- hydroxyethyl)amino) ethyl) amino)methyl)-2,5-dimethoxy tetrahydro-2H-pyran-4-yl sulfate (2j)

Yield: 68\%; IR (KBr) $\left(\mathrm{cm}^{-1}\right)$; 3,458 (NH, str), 3,279 (OH-str), 2,957 (CH-str Ar ring), 1,644 ( $\mathrm{N}=\mathrm{CH}$, str), 1,383 (N-C, str), $1,321(\mathrm{~S}=\mathrm{O}), 812(\mathrm{C}-\mathrm{O}-\mathrm{S}, \mathrm{str}) .{ }^{1} \mathrm{H}$ NMR $\left(\mathrm{DMSO}^{-} \mathrm{d}_{6}\right), \delta(\mathrm{ppm})$ :

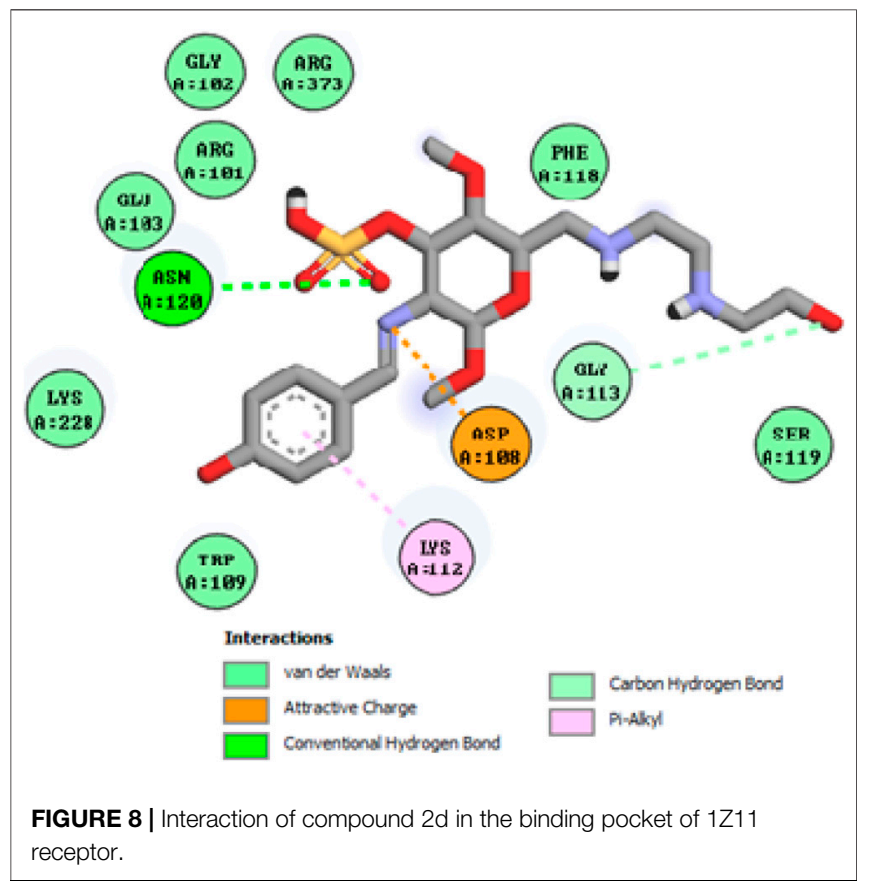

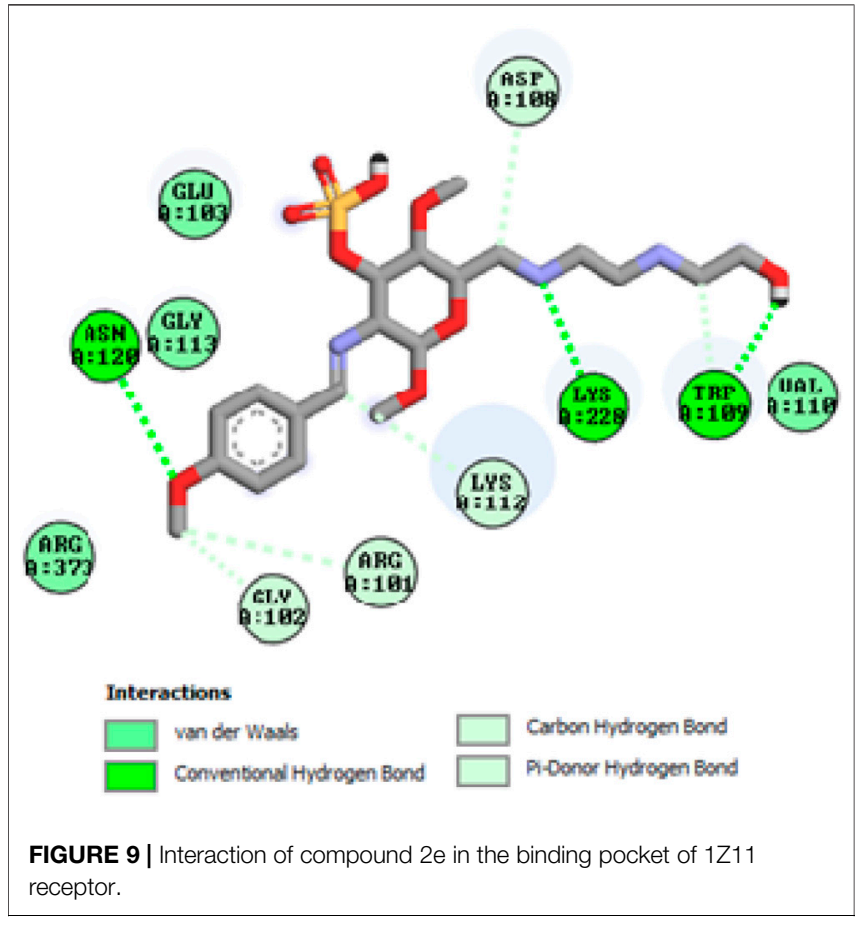

$8.25(\mathrm{~s}, 1 \mathrm{H}, \mathrm{N}=\mathrm{CH}), 7.72-6.50(\mathrm{~m}, 3 \mathrm{H}$, furfural $), 5.26-3.17(\mathrm{~m}$, $5 \mathrm{H}, \mathrm{CS}-\mathrm{H}), 3.68(\mathrm{~s}, 1 \mathrm{H}, \mathrm{OH}), 3.57-3.34\left(\mathrm{~s}, 6 \mathrm{H}, \mathrm{OCH}_{3}-\mathrm{CS}\right)$, $3.43-2.72\left(\mathrm{~m}, 4 \mathrm{H}, \mathrm{OH}-\left(\mathrm{CH}_{2}\right)_{2}\right), 2.81-2.55\left(\mathrm{~m}, 2 \mathrm{H}, \mathrm{CH}_{2}-\mathrm{NH}\right)$, $2.62-2.53\left(\mathrm{~m}, 4 \mathrm{H}, \mathrm{N}\left(\mathrm{CH}_{2}\right)_{2}\right), 2.05(\mathrm{~s}, 2 \mathrm{H}, \mathrm{NH}) ;{ }^{13} \mathrm{C} \mathrm{NMR}$

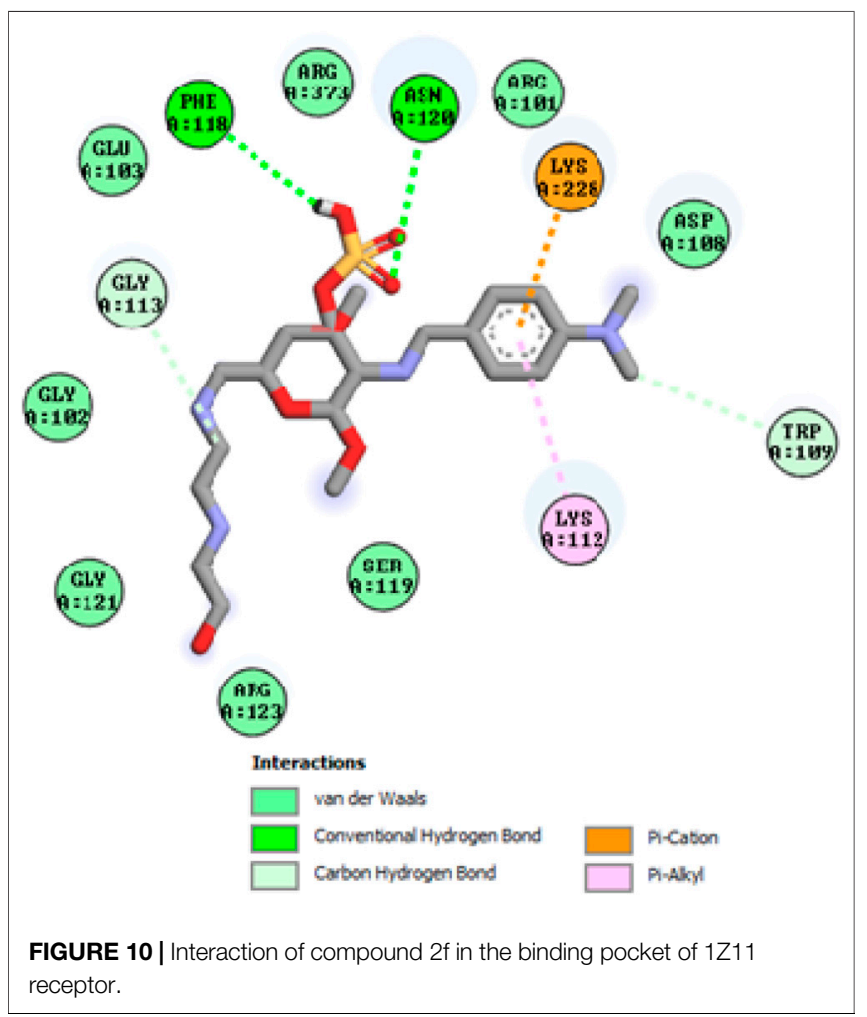




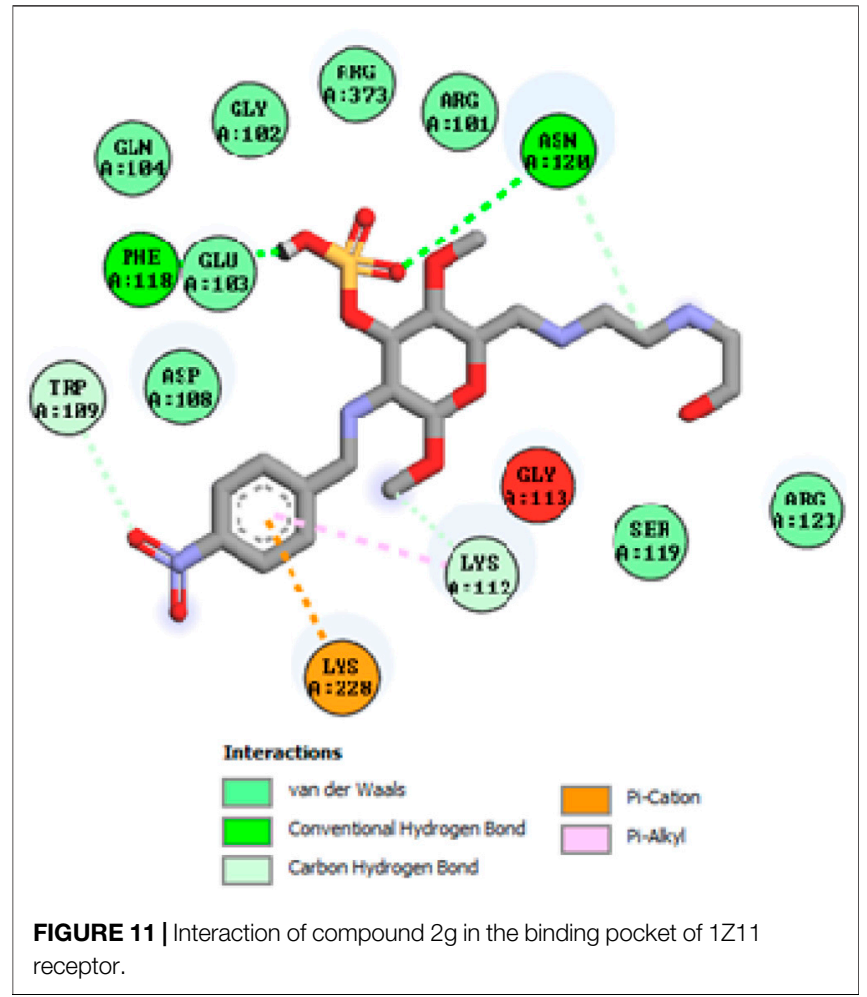

$\left(\mathrm{DMSO}-\mathrm{d}_{6}\right) \delta(\mathrm{ppm}): 163.2(1 \mathrm{C}, \mathrm{N}=\mathrm{CH}), 149.6,144.2,118.3$, 112.9 (4C, furfural), 112.9, 87.8, 71.7, 65.6, 60.5 (5C, CS), 61.4, 52.3 (2C, C-OH), 57.2, 55.1 (2C, $\left.\mathrm{OCH}_{3}-\mathrm{CS}\right), 49.8,49.1$ (2C, N-C in amine), $48.6(1 \mathrm{C}, \mathrm{N}-\mathrm{CH})$.

\section{BIOLOGICAL SCREENING}

\section{Cytotoxic Activity}

The cytotoxic activity of the newly synthesized compounds (2a$2 \mathrm{j}$ ) was tested using a previously described method (Scudiere et al., 1988; Surendra Kumar et al., 2017). Compounds 2a-2j was tested in the MCF-7 cell line for $48 \mathrm{~h}$ using a single dose of primary anticancer assay at a concentration of $100 \mu \mathrm{m}$ (MTT anticancer assay). MCF-7 breast cell line was employed in this study. The MCF-7 cell line was pre-incubated on a micro titer plate in this technique. Each test's results are expressed as a percentage of the treated cells' growth relative to the untreated control cells. Compounds with anticancer activity were those that reduced the proliferation of the cell lines by $30 \%$ or less. $0.1 \mathrm{ml}$ of the cell suspension (containing $5 \times 10^{6}$ cells $/ 100 \mu \mathrm{L}$ ) and $0.1 \mathrm{ml}$ of the test solution $(6.25-100 \mu \mathrm{g}$ in $1 \%$ DMSO such that the final concentration of DMSO in media was less than $1 \%$ ) were added to the 9 well plates and incubated at $37^{\circ} \mathrm{C}$ for $72 \mathrm{~h}$ in a 5 percent $\mathrm{CO}_{2}$ incubator. The control wells included 1 percent DMSO and cell suspension, while the blank contained simply cell suspension. After $72 \mathrm{~h}, 20 \mu \mathrm{L}$ of MTT were added and left in the $\mathrm{CO}_{2}$ incubator for $2 \mathrm{~h}$ before adding $100 \mu \mathrm{L}$ of propanol. To protect the plate from light, it was wrapped in aluminum foil. The 9 well plates were then shaken for $10-25 \mathrm{~min}$ in a rotary shaker. The 9 well plates were

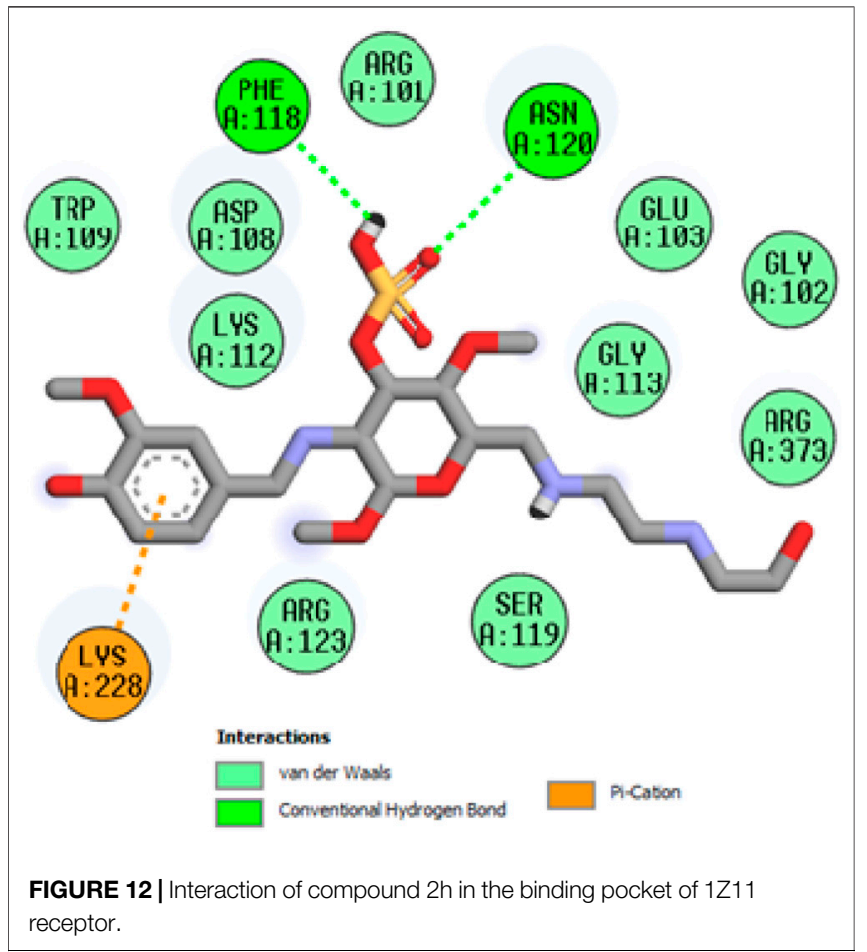

processed on an ELISA reader for absorbance at $562 \mathrm{~nm}$ after 10-20 min.

\section{Molecular Docking}

The binding abilities and interaction between compound $2 \mathrm{a}-2 \mathrm{j}$, Doxorubicin, and the Human microsomal cytochrome P450 2A6 complexed with Methoxsalen (PDB ID: 1Z11) protein were investigated using Autodock vina 1.1.2 (Trott and Olson, 2009). To discover the target protein, the Protein Data Bank (http://www.rcsb.org) was used (PDB ID: 1Z11). The 3D structures of the compounds $2 \mathrm{a}-2 \mathrm{j}$ and Doxorubicin were created using Chem3D Pro 12.0 and Chem Draw Ultra 12.0. The AutoDock Tools 1.5.6 application suite was used to build the input files for Autodock Vina. The grid co-ordinates for the target protein were observed to be $56.316,77.155,60.326$, with size $\mathrm{x}, \mathrm{y}, \mathrm{z}$ : 24, 20, 20 and 1.0 spacing. The interpretation of exhaustiveness has been assigned the number eight. Other Vina docking parameters are enabled by default and are not listed. The compound with the lowest binding affinity value obtained the highest score, which was determined visually using the software Discovery Studio 2019.

\section{RESULT AND DISCUSSION}

The novel chitosan compounds were established by ${ }^{1} \mathrm{H}$ NMR, and ${ }^{13} \mathrm{C}$ NMR, as presented in the Supplementary Materials. The FTIR spectra presented absorption bands $(2 \mathrm{a}-2 \mathrm{j})$ at $3,450-3,458$, 3,280-3,289, 3,050-3,057, 2,928, 1,645, 1,592, 1,474, 1,420, 1,242 and $815 \mathrm{~cm}^{-1}$, confirming the $\mathrm{NH}, \mathrm{OH}, \mathrm{Ar}-\mathrm{H}, \mathrm{CH}, \mathrm{N}=\mathrm{CH}, \mathrm{NH}_{2}$, $(\mathrm{N}-\mathrm{O}), \mathrm{N}-\mathrm{C},(\mathrm{S}=\mathrm{O})$, and $(\mathrm{C}-\mathrm{O}-\mathrm{S})$ groups. The absorption bands 


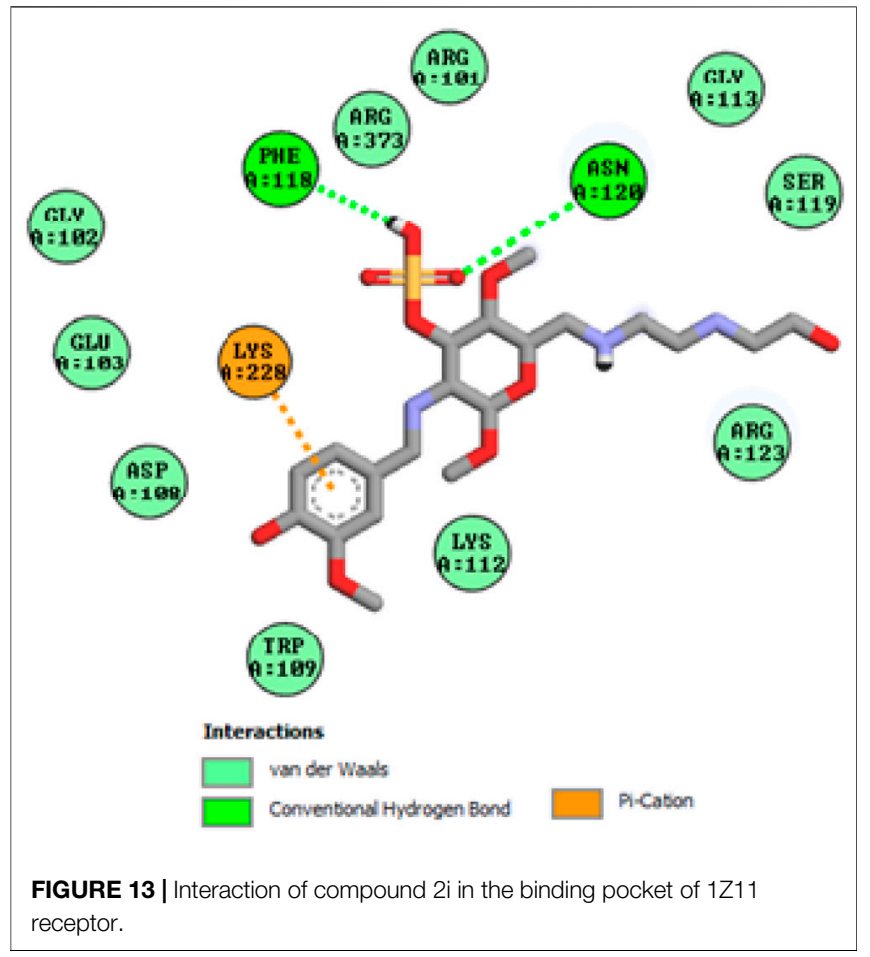

of other compounds are summarized in the Experimental section. The ${ }^{1} \mathrm{H}$ NMR spectra $(2 \mathrm{a}-2 \mathrm{j})$ show shifts in the chemical values at 8.18-8.45, 7.52-7.85, 6.50-7.72,3.64-3.70, 3.33-3.52, 3.10-5.21, $2.72-3.43,2.54-2.80,2.51-2.68$ and $2.02-2.07 \mathrm{ppm}$, confirming the presences of $\mathrm{N}=\mathrm{CH}, \mathrm{Ph}-\mathrm{CH}$, furfural, $\mathrm{CH}_{2}-\mathrm{OH}, \mathrm{OCH}_{3}, \mathrm{CS}-\mathrm{H}$, $\mathrm{OH}-\left(\mathrm{CH}_{2}\right)_{2}, \mathrm{CH}_{2}-\mathrm{NH}, \mathrm{N}\left(\mathrm{CH}_{2}\right)_{2}$, and $\mathrm{NH}$ protons, respectively. The ${ }^{1} \mathrm{H}$ NMR spectra of other compounds are summarized in the Experimental section. The ${ }^{13} \mathrm{C}$ NMR $(2 \mathrm{a}-2 \mathrm{j})$ showed signals at 163.2-165.5, 128.9-136.5, 112.9-149.6, 60.7-112.3, 52.0-61.7, 55.7-57.6, and 48.3-49.0 ppm, confirming the presence of carbon atom in $\mathrm{N}=\mathrm{CH}, \mathrm{Ph}$, furfural, $\mathrm{CS}, \mathrm{C}-\mathrm{OH}, \mathrm{OCH}_{3}, \mathrm{~N}-\mathrm{C}$, and $\mathrm{N}-\mathrm{CH}$. The shift in chemical values of other compounds are given in the experimental section. Compound 2 ( $2.5 \mathrm{~g}$ dissolved in acetic acid with ethanol) and 4-chlorobenzaldehyde $(1.2 \mathrm{ml})$ were mixed with ethanol and stirred for $8 \mathrm{~h}$ at $60^{\circ} \mathrm{C}$. The light-yellow gel obtained indicates the formation of compound $2 \mathrm{~b}$. A solution of $5 \% \mathrm{NaOH}$ was used to precipitate the product, was filtered, and was washed with ice-cold water and ethanol to remove any unreacted products. The final product was soluble in DMF. In this synthesize of chitosan derivatives without using any catalyst, we obtained better yields with in a shorter reaction time. The progress of the reaction was monitored by TLC. The same procedure was followed by the synthesis of other sulfonated chitosan derivatives $2 \mathrm{c}-2 \mathrm{j}$. The synthesized compounds $2 \mathrm{a}-2 \mathrm{j}$ were assessed for their cytotoxic activities against the MCF-7 cancer cell line and against the normal cell lines breast cancer cell MCF-10A and lung cell MRC-5 using an MTT assay, and doxorubicin was used as a standard. Compound $2 \mathrm{~h}$ was found to be highly active, with a $\mathrm{GI}_{50}$ value of $0.02 \mu \mathrm{M}$ for MCF-7 compared with other compounds. The synthesized chitosan derivatives showed less cytotoxicity in normal cell lines MCF-

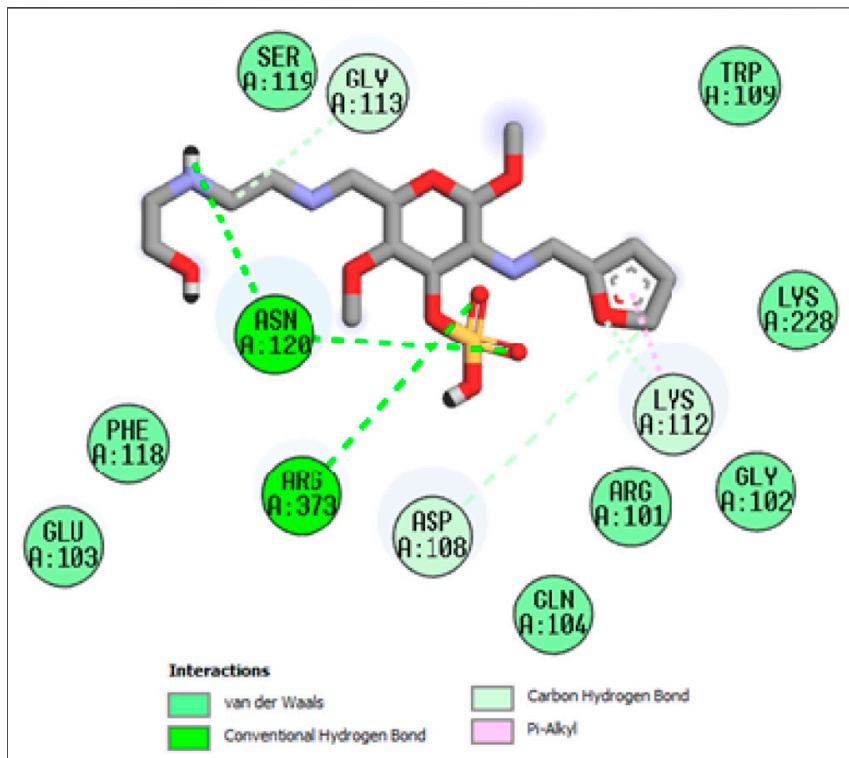

FIGURE 14 | Interaction of compound 2j in the binding pocket of 1 Z11 receptor.

$10 \mathrm{~A}$ and MRC-5, with $\mathrm{IC}_{50}>100 \mathrm{~g} / \mathrm{ml}$, indicating that their use is safe. Scheme 1 illustrates the synthesis of sulfonated chitosan derivatives.

\section{XRD or X-Ray Diffraction study}

An X-Ray diffraction investigation of chitosan derivatives are shown in Figure 2 (Kumar et al., 2009) it shows modest peaks for chitosan derivatives at $2 \theta$ of $20^{\circ}$. In chitosan derivatives the very sharp peaks at $20^{\circ}$ became narrow, it indicates that chitosan derivative has moral compatibility and good development of porous xerogel network. According to the XRD results the chitosan derivative is amorphous in nature.

\section{FTIR spectroscopy of highly Active compound $2 \mathrm{~h}$}

In FTIR spectra of chitosan $\mathrm{NH}$ peak was observed at $3456 \mathrm{~cm}^{-1}$ which can be assigned to stretching vibration of amino group, another peak observed at $3,278 \mathrm{~cm}^{-1}$ can be attributed to hydroxyl group $(\mathrm{OH}, \mathrm{str})$. Similarly the stretching vibration of carbon and hydrogen in aromatic ring observed at $2,949 \mathrm{~cm}^{-1}$. The sharp characteristic peak at $1647 \mathrm{~cm}^{-1} \mathrm{can}$ be attributed to stretching vibration of imine linkage $(\mathrm{N}=\mathrm{CH}$, str). In the chitosan-Schiffbase the stretching vibration of $\mathrm{N}-\mathrm{C}$ observed at $1381 \mathrm{~cm}^{-1}$ can be assigned to $(\mathrm{N}-\mathrm{C}$, str), another sharp peak at $1324 \mathrm{~cm}^{-1}$ can be attributed to stretching vibration of $(\mathrm{S}=\mathrm{O}$, str), and C-O-S can be observed as a stretching vibration of $975 \mathrm{~cm}^{-1}$. The IR spectrum of highly active compound are shown in Figure 3.

\section{Scanning Electron Microscopy}

One of the most effective techniques for studying surface phenomena of prepared materials is the scanning electron microscope (SEM). The morphology of schiffbase 


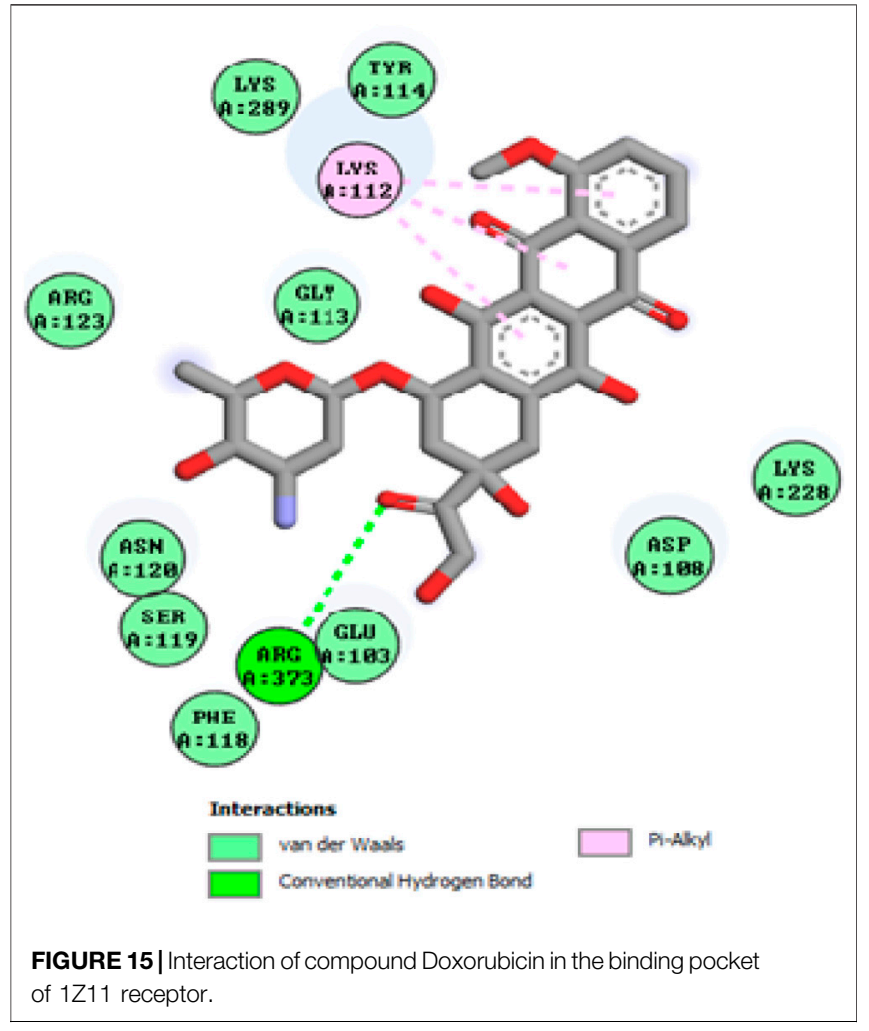

of sulfonated chitosan derivative $2 \mathrm{~h}$ is the particles with sizes of $200 \mathrm{~nm}$. These observations show that the microstructures of chitosan changed after chemical modification. The SEM image of chitosan analogues are shown in Figure 4.

\section{Cytotoxic Activity}

The newly synthesized Schiff base of sulfonated chitosan derivatives (2a-2j) were screened for their cytotoxic activity using MTT anticancer assay (dose of $100 \mu \mathrm{M}$ at $48 \mathrm{~h}$ ) against MCF-7 cancer cell line, andalso evaluated for their possible cytotoxicity in breast cancer cell (MCF-10A), and lung cells (MRC-5) by employing MTT assay. The assay results suggested that these compounds did not significantly affect normal breast, lung cells' growth (As most of the compound's IC50 values are $>100)$. The Table 1 shows the growth inhibitor concentration $\left(\mathrm{GI}_{50}\right)$, total growth of inhibition (TGI), and lethal concentration (LC50) values. Moreover the compound $2 \mathrm{~h}\left(\mathrm{GI}_{50}=\right.$ $0.02 \mu \mathrm{M})$ was relatively more active than standard doxorubicin and other compounds. The in vitro cytotoxicity of Schiff base of sulfonated chitosan derivatives of normal cell lines are given in Table 2. Finally, compound $2 \mathrm{~h}$ can be used as a lead compound to further develop more effective drugs for the MCF-7 (breast) cancer cell line.

\section{Docking studies}

To acquire a better understanding of the future evolution of biotic behavior, docking mock-ups were added. The docking impact of compounds $2 \mathrm{a}-2 \mathrm{j}$ and Doxorubicin regulation with protein $1 \mathrm{Z} 11$ was tested using the Autodock Vina software. Compound $2 \mathrm{~h}$ has a higher binding affinity for $1 \mathrm{Z11}$ protein $(-5.9 \mathrm{kcal} / \mathrm{mol})$ than other compounds and a lower binding affinity for Doxorubicin $(-5.3 \mathrm{kcal} / \mathrm{mol})$. Compound $2 \mathrm{~h}$ makes two hydrogen bonds with

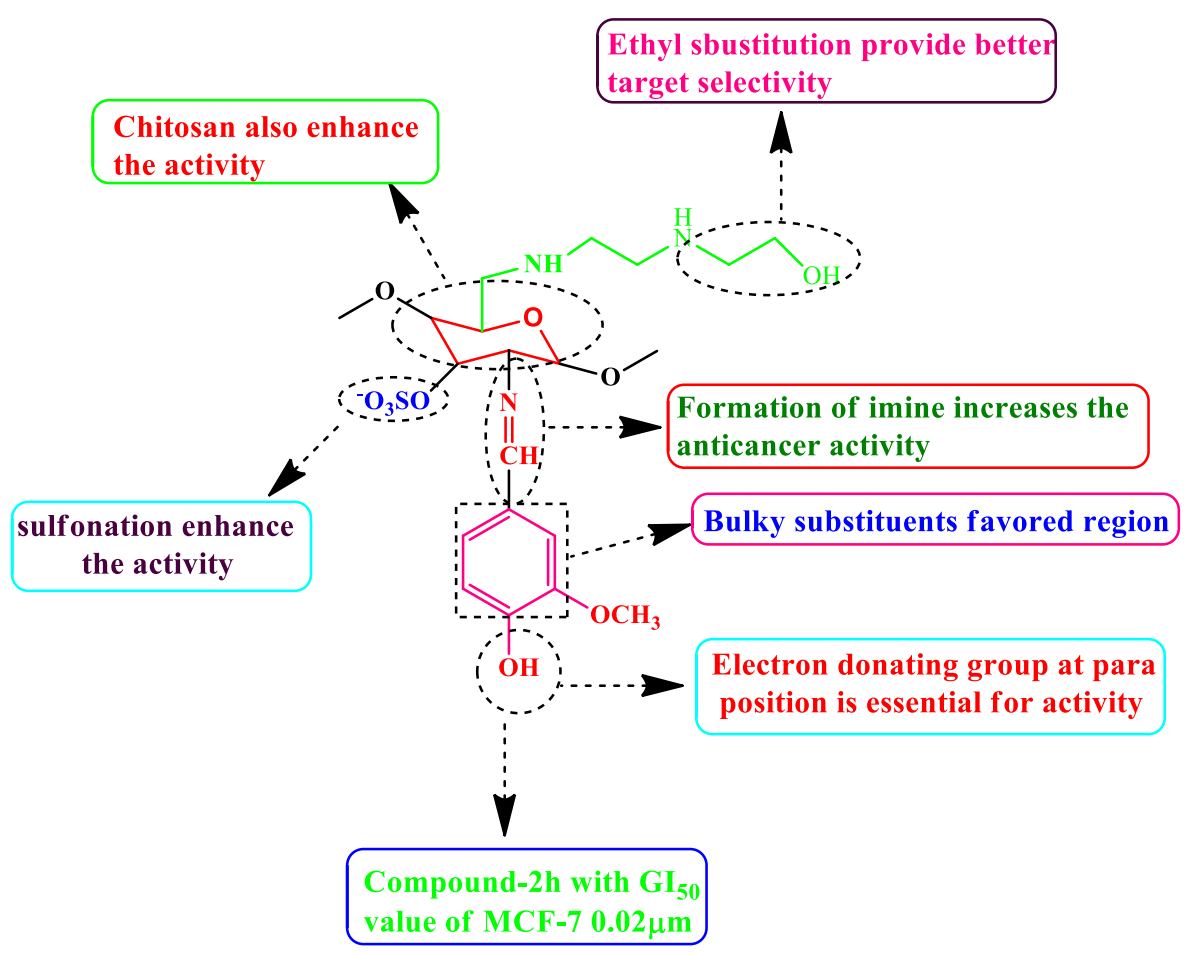

FIGURE 16 | SAR of highly active compound. 
the $1 \mathrm{Z} 11$ receptor. In the hydrogen bonding interaction, the amino acid residues Phe118 and Asn120 were active. In hydrophobic interaction, the amino acid residues Arg101, Gly102, Glu103, Asp108, Trp109, Lys112, Gly113, Ser119, Arg123, Lys228 and Arg373 were active. Doxorubicin regulates the $1 \mathrm{Z11}$ receptor by forming one hydrogen bonds with it. In the hydrogen bonding interaction, the amino acid residues Arg373 were active. The hydrophobic interactions of Glu103, Asp108, Lys112, Gly113, Tyr114, Phe118, Ser119, Asn120, Arg123, Lys228 and Lys289 were observed. Figures 6, 7 show the hydrogen bonding and hydrophobic interactions of amino acid residues in the $1 \mathrm{Z} 11$ protein with the $2 \mathrm{a}-2 \mathrm{j}$ and Doxorubicin compounds, respectively. The results suggest that compound $2 \mathrm{~h}$ has a significant inhibitory effects for Doxorubicin regulation in the target protein. The docking interaction results are present in Table 3. All the docking images are given in Figures 5-15.

\section{Structure Activity Relationship}

The selected functional group plays important role in modifying the cytotoxic activity of compounds within a certain system can be identified via SAR analysis. Preliminary SARs might be analyzed using the cytotoxic activity data of the chitosan derivatives. Compound $2 \mathrm{~h}$ is the most effective (MCF-7, $\mathrm{GI}_{50}=0.02 \mu \mathrm{M}$ ) control doxorubicin among the chitosan derivatives. Owing to the occurrence of chitosan moiety fused to a vanillin, it was revealed that the new compound acquires a strong cytotoxic effect against cancer cell types the presence of electron donating hydroxyl group in para position enhances the activity as well as sulfonation of chitosan also increases the anticancer activity. SAR of highly active compound present in Figure $\mathbf{1 6 .}$

\section{CONCLUSION}

In this paper, we synthesized chitosan derivatives of $2 a-2 j$ that were characterized via FTIR, NMR, and morphology study was carried out by XRD and SEM. The synthesized compounds $2 \mathrm{a}-2 \mathrm{j}$ were assessed for their cytotoxic activities against the MCF-7 cancer cell line, normal cell lines of breast cancer cell MCF-10A and lung cell MRC-5 using an MTT assay. Doxorubicin was used as a standard. Compound $2 \mathrm{~h}$ was

\section{REFERENCES}

Adhikari, H. S., and Yadav, P. N. (2018). Anticancer Activity of Chitosan, Chitosan Derivatives, and Their Mechanism of Action. Int. J. Biomater. 2018, 1-29. doi:10.1155/2018/2952085

Ahmed, S., and Ikram, S. (2016). Chitosan Based Scaffolds and Their Applications in Wound Healing. Achievements Life Sci. 10 (1), 27-37. doi:10.1016/j.als.2016.04.001

Ali, I., Lone, M., Al-Othman, Z., Al-Warthan, A., and Sanagi, M. (2015). Heterocyclic Scaffolds: Centrality in Anticancer Drug Development. Curr. Drug Targets 16 (7), 711-734. doi:10.2174/1389450116666150309115922

Ali, I., Wani, W. A., and Saleem, K. (2011). Cancer Scenario in India with Future Perspectives. Cancer Ther. 8 (1), 56-70.

Ali, S., Sangi, L., Kumar, N., Kumar, B., Khurshid, Z., and Zafar, M. S. (2020). Evaluating Antibacterial and Surface Mechanical Properties of Chitosan Modified Dental Resin Composites. Technol. Health Care 28, 165-173. doi:10.3233/THC-181568 found to be highly active, with a $\mathrm{GI}_{50}$ value of $0.02 \mu \mathrm{M}$ for MCF-7 compared with other compounds. The synthesized chitosan derivatives showed less cytotoxicity in normal cell lines MCF-10A and MRC-5, with $\mathrm{IC}_{50}>100 \mathrm{~g} / \mathrm{ml}$, indicating that their use is safe. Furthermore In silico molecular docking analysis against the 1Z11 receptor has been studied, compound $2 \mathrm{~h}$ has a higher binding affinity for $1 \mathrm{Z11}$ protein $(-5.9 \mathrm{kcal} / \mathrm{mol})$ compared with other compounds and Doxorubicin $(-5.3 \mathrm{kcal} / \mathrm{mol})$. Finally, the compound $2 \mathrm{~h}$ has great activity, and therefore it will be researched for further anticancer drug.

\section{DATA AVAILABILITY STATEMENT}

The datasets presented in this study can be found in online repositories. The names of the repository/repositories and accession number(s) can be found in the article/Supplementary Material.

\section{AUTHOR CONTRIBUTIONS}

PP: Analysis of organic compound and spectrum analysis; PG: organic compounds preparation; DA: provision of study materials; SA: Financial Support; RG: Manuscript editing; AI: All kinds of spectral analysis and RS: Project administration and all aspects of this manuscript's chemistry and biology have been investigated. The manuscript was writing original draft preparation through the contributions of all authors.

\section{FUNDING}

This work was funded by the Researchers Supporting Project number (RSP-2021/27), King Saud University, Riyadh, Saudi Arabia.

\section{SUPPLEMENTARY MATERIAL}

The Supplementary Material for this article can be found online at: https:/www.frontiersin.org/articles/10.3389/fchem.2021.796599/ full\#supplementary-material

Andreadis, C., Vahtsevanos, K., Sidiras, T., Thomaidis, I., Antoniadis, K., and Mouratidou, D. (2003). 5-Fluorouracil and Cisplatin in the Treatment of Advanced Oral Cancer. Oral Oncol. 39 (4), 380-385. doi:10.1016/S1368-8375(02)00141-0

Aranaz, I., Harris, R., and Heras, A. (2010). Chitosan Amphiphilic Derivatives. Chemistry and Applications. Curr. Org. Chem. 14 (3), 308-330. doi:10.2174/138527210790231919

Bakar, L. M., Abdullah, M. Z., Doolaanea, A. A., and Ichwan, S. J. A. (2017). PLGAchitosan Nanoparticle-Mediated Gene Delivery for Oral Cancer Treatment: a Brief Review. J. Phys. Conf. Ser. 884, 012117. doi:10.1088/1742-6596/884/1/012117

Bray, F., Ferlay, J., Soerjomataram, I., Siegel, R. L., Torre, L. A., and Jemal, A. (2018). Global Cancer Statistics 2018: GLOBOCAN Estimates of Incidence and Mortality Worldwide for 36 Cancers in 185 Countries. CA: A Cancer J. Clinicians 68, 394-424. doi:10.3322/caac.21492

Bray, F., and Møller, B. (2006). Predicting the Future burden of Cancer. Nat. Rev. Cancer 6 (1), 63-74. doi:10.1038/nrc1781

Chen, G., and Gong, R. (2016). Study on Fluorouracil-Chitosan Nanoparticle Preparation and its Antitumor Effect. Saudi Pharm. J. 24, 250-253. doi:10.1016/j.jsps.2016.04.008 
Cheung, R., Ng, T., Wong, J., and Chan, W. (2015). Chitosan: an Update on Potential Biomedical and Pharmaceutical Applications. Mar. Drugs 13 (8), 5156-5186. doi:10.3390/md13085156

Husain, S., Al-Samadani, K. H., Najeeb, S., Zafar, M. S., Khurshid, Z., Zohaib, S., et al. (2017). Chitosan Biomaterials for Current and Potential Dental Applications. Materials 10, 602. doi:10.3390/ma10060602

Imran, M., Sajwan, M., Alsuwayt, B., and Asif, M. (2020). Synthesis, Characterization and Anticoagulant Activity of Chitosan Derivatives. Saudi Pharm. J. 28, 25-32. doi:10.1016/j.jsps.2019.11.003

Jiang, M., Ouyang, H., Ruan, P., Zhao, H., Pi, Z., Huang, S., et al. (2011). Chitosan Derivatives Inhibit Cell Proliferation and Induce Apoptosis in Breast Cancer Cells. Anticancer Res. 31, 1321-1328.

Kim, S. (2018). Competitive Biological Activities of Chitosan and its Derivatives: Antimicrobial, Antioxidant, Anticancer, and Anti-inflammatory Activities. Int. J. Polym. Sci. 2018, 1-13. doi:10.1155/2018/1708172

Kumar, S., Dutta, J., and Dutta, P. K. (2009). Preparation and Characterization of N-Heterocyclic Chitosan Derivative Based Gels for Biomedical Applications. Int. J. Biol. Macromolecules 45, 330-337. doi:10.1016/j.ijbiomac.2009.08.002

Kumirska, J., Weinhold, M. X., Thöming, J., and Stepnowski, P. (2011). Biomedical Activity of Chitin/Chitosan Based Materials-Influence of Physicochemical Properties Apart from Molecular Weight and Degree of N-Acetylation. Polymers 3 (4), 1875-1901. doi:10.3390/polym3041875

Kuppusamy, S., and Karuppaiah, J. (2013). Screening of Antiproliferative Effect of Chitosan on Tumor Growth and Metastasis in T24 Urinary Bladder Cancer Cell Line. Austrl-Asian J. ofCancer. 12 (3), 145-149.

Li, Q., Li, Q., Tan, W., Zhang, J., and Guo, Z. (2020). Phenolic-containing Chitosan Quaternary Ammonium Derivatives and Their Significantly Enhanced Antioxidant and Antitumor Properties. Carbohydr. Res. 498, 108169. doi:10.1016/j.carres.2020.108169

Li, Z., Yang, F., and Yang, R. (2015). Synthesis and Characterization of Chitosan Derivatives with Dual-Antibacterial Functional Groups. Int. J. Biol. Macromolecules 75, 378-387. doi:10.1016/j.ijbiomac.2015.01.056

Mahmood, M. A., Madni, A., Rehman, M., Rahim, M. A., and Jabar, A. (2019). Ionically Cross-Linked Chitosan Nanoparticles for Sustained Delivery of Docetaxel: Fabrication, Post-Formulation and Acute Oral Toxicity Evaluation. Int. J. Nanomedicine 14, 10035-10046. doi:10.2147/IJN.S232350

Martins, C. R., Ruggeri, G., and De Paoli, M.-A. (2003). Synthesis in Pilot Plant Scale and Physical Properties of Sulfonated Polystyrene. J. Braz. Chem. Soc. 14, 797-802. doi:10.1590/S0103-50532003000500015

Morrison, R. T., and Boyd, R. N. (1997). Organic Chemistry. Sixth ed. Hoboken, NJ, USA: Prentice-Hall, 517-527.

Muanprasat, C., and Chatsudthipong, V. (2017). Chitosan Oligosaccharide: Biological Activities and Potential Therapeutic Applications. Pharmacol. Ther. 170, 80-97. doi:10.1016/j.pharmthera.2016.10.013

Pereira, C., Leao, M., Soares, J., Bessa, C., and Saraiva, L. (2012). New Therapeutic Strategies for Cancer and Neurodegeneration Emerging from Yeast Cell-Based Systems. Curr. Pharm. Des. 18 (27), 4223-4235. doi:10.2174/138161212802430422

Perni, S., and Prokopovich, P. (2017). Poly-beta-amino-esters Nano-Vehicles Based Drug Delivery System for Cartilage. Nanomedicine: Nanotechnology, Biol. Med. 13, 539-548. doi:10.1016/j.nano.2016.10.001

Qasim, S., Zafar, M., Najeeb, S., Khurshid, Z., Shah, A., Husain, S., et al. (2018). Electrospinning of Chitosan-Based Solutions for Tissue Engineering and Regenerative Medicine. Int. J. Mol. Sci. 19, 407. doi:10.3390/ijms19020407

Saeed, R. M., Dmour, I., and Taha, M. O. (2020). Stable Chitosan-Based Nanoparticles Using Polyphosphoric Acid or Hexametaphosphate for Tandem Ionotropic/ Covalent Crosslinking and Subsequent Investigation as Novel Vehicles for Drug Delivery. Front. Bioeng. Biotechnol. 8, 4. doi:10.3389/fbioe.2020.00004
Sarwar, M. S., Huang, Q., Ghaffar, A., Abid, M. A., Zafar, M. S., Khurshid, Z., et al. (2020). A Smart Drug Delivery System Based on Biodegradable Chitosan/Poly(allylamine Hydrochloride) Blend Films. Pharmaceutics 12, 131. doi:10.3390/pharmaceutics12020131

Scudiere, D. A., Shoemaker, R. H., Paul, K. D., Monks, A., Tierney, S., Nofziger, T. H., et al. (1988). Evaluation of a Soluble Tetrazolium/Formazan Assay for Cell Growth and Drug Sensitivity in Culture Using Human and Other Tumor Cell Lines. Cancer Res. 48, 4827-4833.

Srivastava, S., Koay, E. J., Borowsky, A. D., De Marzo, A. M., Ghosh, S., Wagner, P. D., et al. (2019). Cancer Overdiagnosis: a Biological challenge and Clinical Dilemma. Nat. Rev. Cancer 19, 349-358. doi:10.1038/s41568019-0142-8

Surendra Kumar, R., Moydeen, M., Al-Deyab, S. S., Manilal, A., and Idhayadhulla, A. (2017). Synthesis of New Morpholine - Connected Pyrazolidine Derivatives and Their Antimicrobial, Antioxidant, and Cytotoxic Activities. Bioorg. Med. Chem. Lett. 27, 66-71. doi:10.1016/j.bmcl.2016.11.032

Taher, F. A., Ibrahim, S. A., El-Aziz, A. A., Abou El-Nour, M. F., El-Sheikh, M. A., ElHusseiny, N., et al. (2019). Anti-Proliferative Effect of Chitosan Nanoparticles (Extracted from Crayfish Procambarus Clarkii, Crustacea: Cambaridae) against MDA-MB-231 and SK-BR-3 Human Breast Cancer Cell Lines. Int. J. Biol. Macromolecules 126, 478-487. doi:10.1016/j.ijbiomac.2018.12.151

Trott, O., and Olson, A. J. (2009). AutoDock Vina: Improving the Speed and Accuracy of Docking with a New Scoring Function, Efficient Optimization, and Multithreading. J. Comput. Chem. 31, 455. doi:10.1002/jcc.21334

Wang, P., He, H., Cai, R., Tao, G., Yang, M., Zuo, H., et al. (2019). Cross-linking of Dialdehyde Carboxymethyl Cellulose with Silk Sericin to Reinforce Sericin Film for Potential Biomedical Application. Carbohydr. Polym. 212, 403-411. doi:10.1016/j.carbpol.2019.02.069

Wimardhani, Y. S., F. Suniarti, D., J. Freisleben, H., Wanandi, S. I., C. Siregar, N., and Ikeda, M.-A. (2014). Chitosan Exerts Anticancer Activity through Induction of Apoptosis and Cell Cycle Arrest in Oral Cancer Cells. J. Oral Sci. 56 (2), 119-126. doi:10.2334/josnusd.56.119

Zhang, M., Li, X. H., Gong, Y. D., Zhao, N. M., and Zhang, X. F. (2002). Properties and Biocompatibility of Chitosan Films Modified by Blending with PEG. Biomaterials 23 (13), 2641-2648. doi:10.1016/S0142-9612(01) 00403-3

Zhang, Y., Sun, T., and Jiang, C. (2018). Biomacromolecules as Carriers in Drug Delivery and Tissue Engineering. Acta Pharmaceutica Sinica B 8 (1), 34-50. doi:10.1016/j.apsb.2017.11.005

Conflict of Interest: The authors declare that the research was conducted in the absence of any commercial or financial relationships that could be construed as a potential conflict of interest.

Publisher's Note: All claims expressed in this article are solely those of the authors and do not necessarily represent those of their affiliated organizations, or those of the publisher, the editors and the reviewers. Any product that may be evaluated in this article, or claim that may be made by its manufacturer, is not guaranteed or endorsed by the publisher.

Copyright (C) 2022 Packialakshmi, Gobinath, Ali, Alarifi, Gurusamy, Idhayadhulla and Surendrakumar. This is an open-access article distributed under the terms of the Creative Commons Attribution License (CC BY). The use, distribution or reproduction in other forums is permitted, provided the original author(s) and the copyright owner(s) are credited and that the original publication in this journal is cited, in accordance with accepted academic practice. No use, distribution or reproduction is permitted which does not comply with these terms. 